\title{
Analog-Digital Beamforming in the MU-MISO Downlink by use of Tunable Antenna Loads
}

\author{
Ang Li, Student Member, IEEE, Christos Masouros, Senior Member, IEEE, and Mathini Sellathurai, Senior \\ Member, IEEE
}

\begin{abstract}
We investigate the performance of multi-user multiple-input-single-output (MU-MISO) downlink in the presence of the mutual coupling effect at the transmitter. Contrary to traditional approaches that aim at eliminating this effect, in this paper we propose a joint analog-digital (AD) beamforming scheme that exploits this effect to further improve the system performance. A jointly optimal AD beamformer is firstly obtained by iteratively maximizing the minimum received signalto-interference-plus-noise ratio (SINR) in the digital domain, followed by an optimization on the load impedance of each antenna element in the analog domain. We further introduce a decoupled low-complexity approach, with which existing closedform beamforming schemes can also be efficiently applied. For the consideration of hardware imperfections in practice, we study the case where the analog load values are quantized, and propose a sequential search scheme based on greedy algorithm to efficiently obtain the desired quantized load values. Moreover, we also investigate the imperfect channel state information (CSI) scenarios, where we prove the optimality for closed-form beamformers, and further propose the robust schemes for two typical CSI error models. Simulation results show that with the proposed schemes the mutual coupling effect can be exploited to further improve the performance for both perfect CSI and imperfect CSI.
\end{abstract}

Index Terms-MIMO, beamforming, mutual coupling, optimization, imperfect CSI.

\section{INTRODUCTION}

$\mathbf{T}$ HE widely acknowledged benefits of multiple-inputmultiple-output (MIMO) systems have made them a standard in current and future communication systems. Among MIMO techniques, transmit processing schemes that can transfer the complexity from the user end to the base stations (BSs) have been widely studied and adopted in the latest communication standards [1][2]. In [3], the capacity achieving dirty paper coding (DPC) has been proposed, which is however difficult to implement due to the impractical assumption of

Manuscript received February 28, 2017; revised August 09, 2017, and November 15, 2017; accepted November 18, 2017. The associate editor coordinating the review of this paper and approving it for publication was Prof. R. Dinis. (Corresponding author: Ang Li.)

Copyright (c) 2015 IEEE. Personal use of this material is permitted. However, permission to use this material for any other purposes must be obtained from the IEEE by sending a request to pubs-permissions@ieee.org.

A. Li and C. Masouros are with the Dept. of Electronic and Electrical Eng., University College London, Torrington Place, London, WC1E 7JE, UK (e-mail: ang.li.14@ucl.ac.uk, chris.masouros@ieee.org). M. Sellathurai is with the Signal Processing for Intelligent Systems and Communications Research Group, Heriot-Watt University, EH14 4AS, UK (email: M.Sellathurai@hw.ac.uk)

This work was supported by the Royal Academy of Engineering, UK, the Engineering and Physical Sciences Research Council (EPSRC) project EP/M014150/1, EP/M014126/1, and the China Scholarship Council (CSC). infinite length of codewords and high complexity. To retain the performance benefits of DPC, non-linear techniques known as Tomlinson-Harashima precoding (THP) and vector perturbation (VP) have been introduced [4]-[10], which can offer a close-to-optimal performance. Nevertheless, the sophisticated sphere-search algorithms make the non-linear approaches highly computationally expensive, which makes their applications prohibitive in practice [11]. Therefore, studies on low-complexity linear approaches based on zero-forcing (ZF) have gained research attention and become increasingly popular [12]-[14]. On the other hand, transmit beamforming schemes based on optimization have drawn increasing research attention recently [15]-[17]. One form of the optimization based beamforming is to minimize the total transmit power subject to a minimum signal-to-interference-plus-noise-ratio (SINR) requirement [15]. An alternative downlink beamforming scheme targets at maximizing the minimum SINR subject to a total transmit power constraint [16]. It has been proven in [16][17] that these two optimization problems are inverse problems, and algorithms based on iterative designs and semidefinite programming (SDP) have been proposed to efficiently solve the optimization problems. A symbol-level beamforming scheme is proposed in [18] to further improve the performance by exploiting the constructive interference.

Many existing beamforming schemes are studied with the assumption that BSs are equipped with ideal antenna arrays, where there is no spatial correlation or mutual coupling effect among antenna elements. This is achieved by antenna arrays with the antenna spacing larger than half of the carrier wavelength in practice. Small-scale MIMO BSs such as picocells or femtocells for heterogeneous networks and small access points (APs) for the internet of things (IoTs) however, usually have strict size constraints, which leads to a much reduced antenna spacing. In such cases, the spatial correlation and mutual coupling among antenna elements cannot be ignored [19]-[21]. The effect of the transmit spatial correlation has been studied experimentally in [20][22], and the analysis of the correlation effect on the system performance can be found in [23]-[26]. Designs of the precoders for correlated channels are studied in [27]-[29]. For the mutual coupling effect, in [30] the model of mutual coupling matrix is derived analytically based on a $2 \times 2$ MIMO system and its impact on the system capacity is also studied. The impact of mutual coupling effect is further studied in [31]-[34]. In [31], the effect of mutual coupling on the performance of a beamforming transmission for MIMO under Rician channel conditions is investigated. In [32] it is shown that the mutual coupling effect degrades the 
uplink rate performance when the antenna spacing decreases. The studies in the coupling effect on the channel capacity with respect to the antenna spacing can be found in [35]-[38]. The above studies show that for the performance of MIMO systems, the mutual coupling can be beneficial in some cases while detrimental in some other cases.

To alleviate the performance loss when the mutual coupling effect is detrimental, a number of compensation techniques have been proposed [39]-[44]. In [39], the coupling effect is well alleviated by forming a compensation matrix at the receiver side, whose effectiveness is validated experimentally. In [40], a mutual coupling compensation matrix is derived based on the generalized scattering matrix of the antenna array. [41] proposes a novel structure to suppress the mutual coupling effect by adding a U-shaped microstrip, and has tested its effectiveness. In [42], the mutual coupling effect at low-terahertz (THz) frequencies is studied, and a mantle cloaking method is applied to reduce the mutual coupling between strip dipole antennas. In [43], the calculation of the coupling compensation matrices is given with the knowledge of active element patterns or the measurements of spherical mode expansion. Nevertheless, it should be noted that most of these existing approaches apply to specific scenarios and are not from a signal processing perspective. Moreover, existing studies and techniques on the coupling effect discussed above are aimed at eliminating and minimizing this effect. Different from the above approaches, we introduce a mutual coupling exploitation technique in [45] based on the constructive interference, where it is shown that the coupling effect can be beneficial with by use of tunable antenna loads. Nevertheless, the techniques in [45] only apply to single-user MIMO systems.

To extend the mutual coupling exploitation to multi-user transmission, in this paper we propose a joint analog-digital (AD) optimization-based beamforming scheme which exploits the mutual coupling effect rather than eliminating this effect. Different from conventional $\mathrm{AD}$ techniques in large-scale MIMO systems that target at complexity reduction [46]-[49], the proposed $\mathrm{AD}$ beamforming aims to further improve the performance of compact MIMO systems. In the proposed beamforming scheme, each antenna element is equipped with a tunable load (for example a varactor) to facilitate ana$\log$ processing. In the digital domain, transmit beamforming schemes are considered, while in the analog domain, the mutual coupling matrix is controlled by the tunable loads. By judiciously selecting the value of each antenna load through an iterative approach, the mutual coupling matrix and the beamforming vectors are jointly optimized, which leads to an improved performance. For complexity consideration, we further propose a decoupled low-complexity approach to alleviate the computational burden of the joint scheme, where the digital beamforming vectors are firstly obtained, based on which the optimization on the tunable loads is performed. The decoupled approach also enables the mutual coupling exploitation upon existing closed-form digital beamforming schemes, where the resulting optimization is only applied in the analog domain and the complexity can be further reduced. Furthermore, since in real systems it may be infeasible to obtain any continuous load values due to the imperfection in the hardware components, a more practical case with quantized tunable loads on the optimization of the mutual coupling is considered, where we propose a sequential search scheme based on greedy algorithm to efficiently obtain the desired quantized load values.

Our study is further extended to imperfect CSI, where we consider both the statistical CSI error model and the norm bounded CSI error model. By calculating the SINR of the received symbols, we show that for closed-form beamforming techniques in the digital domain, the load values obtained with perfect CSI can be directly applied to imperfect CSI and the optimality still holds. We then propose the robust schemes for these two CSI error scenarios, where for the statistical CSI errors the robust scheme is designed by incorporating the equivalent noise power, while for the norm bounded CSI errors the robust scheme is designed by considering the worstcase SINR. The implementation of the proposed schemes in practice is also discussed. Simulation results show that both the joint beamforming scheme and the decoupled beamforming scheme outperform the conventional digital beamforming schemes with fixed mutual coupling effect, in both perfect CSI and imperfect CSI scenarios. Accordingly, the profound conclusion from our work is that, hardware imperfections in the form of mutual coupling can be beneficial and can be exploited to further improve the system performance.

For reasons of clarity we summarize the contributions of the paper as:

1) We construct a joint $\mathrm{AD}$ beamforming scheme that exploits the mutual coupling effect to further improve the system performance. We formulate the problem into convex optimization and obtain the optimal beamforming vectors and load impedance value for each antenna array.

2) We further propose a decoupled scheme to alleviate the high complexity of the joint scheme, where we first design the digital-only beamformers and then optimize the load values. For the case where the load values are quantized, we propose a sequential search scheme based on a greedy algorithm to exploit the mutual coupling and efficiently obtain the solution.

3) We discuss the implementation of the proposed schemes in practice regarding the decomposition of the CSI, based on which we analyze the system performance in terms of the received SINR for imperfect CSI. We show that for closed-form beamformers, the proposed schemes can be directly extended to imperfect CSI and the optimality still holds. We also discuss the impedance tuning techniques that enable the fast tuning of the load impedances.

4) We propose the robust SINR optimization for imperfect CSI, where for the statistical CSI errors the robust scheme is designed by incorporating the equivalent noise power, and for the norm bounded CSI errors, the worst-case SINR is considered.

The rest of the paper is organized as follows: Section II introduces the downlink system model. Section III introduces the joint AD beamforming scheme, and in Section IV the decoupled scheme and sequential search scheme are proposed for practical consideration. Section V presents the acquisition of the CSI and the imperfect CSI model. The performance 


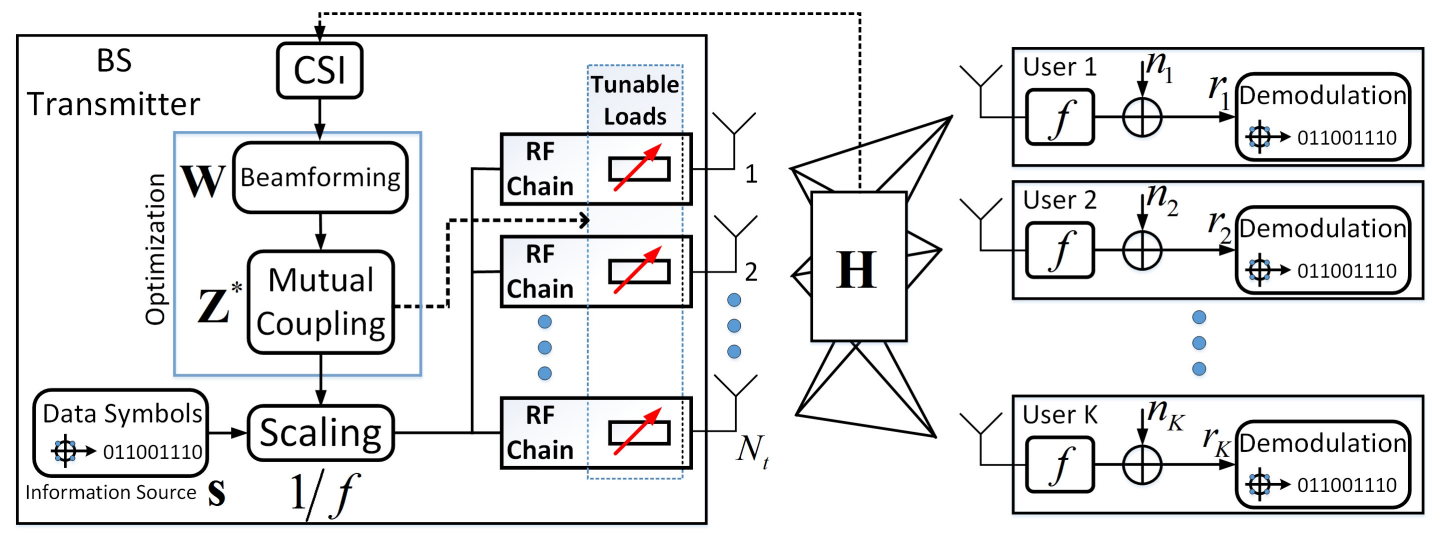

Fig. 1: Block diagram of the system model

metrics are presented in Section VI, based on which the optimality of the closed-form beamformers for imperfect CSI is shown, and in Section VII we propose the robust scheme for imperfect CSI. Section VIII gives a brief discussion on the practical implementation of the proposed schemes. Section IX shows the simulation results and Section $\mathrm{X}$ concludes our paper.

Notations: $a$, a, and $\mathbf{A}$ denote scalar, vector and matrix, respectively. $\mathbb{E}\{\cdot\}, \operatorname{rank}(\cdot),(\cdot)^{H},(\cdot)^{-1}$, and $\operatorname{tr}\{\cdot\}$ denote expectation, rank, conjugate transpose, inverse and trace of a matrix respectively. $|\cdot|$ represents the modulus, $\|\cdot\|$ denotes the Frobenius norm, and $\mathbf{I}$ is the identity matrix. We denote $\mathbf{0}$ as a zero matrix or vector, and $\mathbf{I}_{k}$ denotes the $i$-th column of the diagonal matrix I. $\mathcal{C}^{n \times n}$ represents an $n \times n$ matrix in the complex set, and $\operatorname{diag}(\cdot)$ denotes the conversion of a vector into a diagonal matrix. $\Re(\cdot)$ and $\Im(\cdot)$ denote the real and imaginary part of a complex variable, respectively. $[\mathbf{R}]_{m, n}$ denotes the element of the $m$ th row and $n$th column in $\mathbf{R}$, and $\mathbf{R} \succeq 0$ means that $\mathbf{R}$ is Hermitian positive semi-definite.

\section{SYSTEM MODEL}

\section{A. Downlink System Model}

We consider a multiuser MISO downlink system as shown in Fig. 1, where a BS with $N_{t}$ antennas each with its own radio frequency (RF) chain and tunable load communicates with $K$ single-antenna users simultaneously and we assume $K \leq N_{t}$. Assuming a transmit beamforming matrix $\mathbf{P} \in \mathcal{C}^{N_{t} \times K}$ at the transmitter, the received signal vector after the channel can then be obtained as

$$
\mathbf{y}=\mathbf{H x}+\mathbf{n}=\mathbf{H P s}+\mathbf{n},
$$

where $\mathbf{s} \in \mathcal{C}^{K \times 1}$ is the symbol vector and for simplicity we assume each element in $\mathbf{s}$ is from a normalized constellation and $\mathbb{E}\left\{\mathbf{s s}^{H}\right\}=\mathbf{I}$. $\mathbf{H} \in \mathcal{C}^{K \times N_{t}}$ is the channel matrix and $\mathbf{n}=\left[n_{1}, n_{2}, \ldots, n_{K}\right]^{T}$ is the additive white Gaussian noise (AWGN) vector that follows the complex Gaussian distribution with zero mean. By denoting $\mathbf{H}=\left[\mathbf{h}_{1}^{T}, \mathbf{h}_{2}^{T}, \ldots, \mathbf{h}_{K}^{T}\right]^{T}$ and $\mathbf{P}=\left[\mathbf{p}_{1}, \mathbf{p}_{2}, \ldots, \mathbf{p}_{K}\right]$, the received signal for the $k$ th user can be obtained based on (1) as

$$
y_{k}=\mathbf{h}_{k} \mathbf{P s}+n_{k}=\mathbf{h}_{k} \mathbf{p}_{k} s_{k}+\mathbf{h}_{k} \sum_{i \neq k} \mathbf{p}_{i} s_{i}+n_{k} .
$$

Based on (2), we can express the received SINR of the $k$ th user as

$$
\gamma_{k}=\frac{\left|\mathbf{h}_{k} \mathbf{p}_{k}\right|^{2}}{\sum_{i \neq k}\left|\mathbf{h}_{k} \mathbf{p}_{i}\right|^{2}+\sigma^{2}},
$$

where $\sigma^{2}$ is the noise power. Perfect CSI is firstly assumed, and the effect of imperfect CSI is investigated in the following.

\section{B. Channel Model with Spatial Correlation and Mutual Cou- pling}

We assume a geometric semi-correlated non-line-of-sight (NLOS) Rayleigh flat-fading channel, where the correlation and mutual coupling is considered at the transmitter side [19][20]. We therefore model the $1 \times N_{t}$ channel vector $\mathbf{h}_{k}$ for the $k$-th user as

$$
\mathbf{h}_{k}=\mathbf{g}_{k} \mathbf{A}_{k} \mathbf{Z}
$$

where $\mathbf{g}_{k} \in \mathcal{C}^{1 \times M}$ and $M$ denotes the number of directions of departure (DoDs). We denote each element in $\mathbf{g}_{k}$ as $\mathbf{g}_{k}(i)$ and assume each $\mathbf{g}_{k}(i) \sim \mathcal{C N}(0,1)$, which forms the Rayleigh component of the channel. The mutual coupling effect is fully characterized by the mutual coupling matrix $\mathbf{Z}$, which will be introduced in detail below. $\mathbf{A}_{k} \in \mathcal{C}^{M \times N_{t}}$ represents the transmit-side steering matrix containing $M$ steering vectors of the transmit antenna array. For uniform linear arrays (ULAs), as assumed in this paper, $\mathbf{A}_{k}$ can be modelled as

$$
\mathbf{A}_{k}=\left[\mathbf{a}^{T}\left(\theta_{k, 1}\right), \ldots, \mathbf{a}^{T}\left(\theta_{k, M}\right)\right]^{T} .
$$

In (5), $\mathbf{a}\left(\theta_{k, i}\right) \in \mathcal{C}^{1 \times N_{t}}$ is given by

$$
\mathbf{a}\left(\theta_{k, i}\right)=\left[1, e^{j 2 \pi d \sin \theta_{k, i}}, \ldots, e^{j 2 \pi\left(N_{t}-1\right) d \sin \theta_{k, i}}\right],
$$

where $d$ denotes the equidistant antenna spacing normalized by the carrier wavelength at the BS. $\theta_{k, i}$ denotes the angle of departure (AoDs) and throughout the paper we assume each $\theta_{k, i}$ follows a uniform distribution in $[-\varphi, \varphi]$ [19].

Based on [30][33], we can express the mutual coupling matrix of an antenna array with tunable loads as

$$
\mathbf{Z}\left(\mathbf{z}_{L}\right)=\left[z_{A} \cdot \mathbf{I}+\operatorname{diag}\left(\mathbf{z}_{L}\right)\right]\left[\boldsymbol{\Gamma}+\operatorname{diag}\left(\mathbf{z}_{L}\right)\right]^{-1},
$$

where $z_{A}$ denotes the antenna impedance. $\mathbf{z}_{L}=$ $\left[z_{L_{1}}, z_{L_{2}}, \ldots, z_{L_{N_{t}}}\right]^{T}$ is the tunable load impedance vector 
which we aim to optimize. $\boldsymbol{\Gamma}$ is the mutual impedance matrix and can be expressed as

$$
\boldsymbol{\Gamma}=\left[\begin{array}{ccccc}
z_{A} & z_{m_{1}} & z_{m_{2}} & \cdots & z_{m_{N_{t}-1}} \\
z_{m_{1}} & z_{A} & z_{m_{1}} & \ddots & \vdots \\
z_{m_{2}} & z_{m_{1}} & \ddots & \ddots & z_{m_{2}} \\
\vdots & \ddots & \ddots & \ddots & z_{m_{1}} \\
z_{m_{N_{t}-1}} & \cdots & z_{m_{2}} & z_{m_{1}} & z_{A}
\end{array}\right]
$$

In (7) and (8), $z_{m_{k}}$ denotes the mutual impedance of two antenna elements with the distance of $k d$. In this paper we assume a uniform dipole antenna array, where the calculation of the antenna impedance $z_{A}$ and each mutual impedance $z_{m_{k}}$ can be obtained by the induced electromagnetic-field (EMF) methods based on $d$ (see Appendix).

\section{Proposed Joint BeAmforming Scheme}

In this section we introduce the proposed joint analog-digital beamforming scheme. We note that the joint optimization can offer a better performance at the cost of relatively high computational complexity. Therefore, it is more suitable for low-dimensional problems or as a performance benchmark.

With a tunable load (for example a varactor) employed for each antenna element, the mutual coupling effect can be controlled by modifying the value of each load, as observed in (7). We then jointly optimize the transmit beamformers in the digital domain and the mutual coupling matrix in the analog domain, such that the resulting beamforming vectors and load impedances are jointly optimal, which therefore improves the system performance. To exploit the mutual coupling effect, we first rewrite $\mathbf{H}=\mathbf{D Z}$, where $\mathbf{D}$ is given by

$$
\mathbf{D}=\left[\mathbf{d}_{1}^{T}, \ldots, \mathbf{d}_{k}^{T}, \ldots, \mathbf{d}_{K}^{T}\right]^{T},
$$

and $\mathbf{d}_{k}=\mathbf{g}_{k} \mathbf{A}_{k}$. Then, (1) can be rewritten as

$$
\mathbf{y}=\mathbf{D Z P s}+\mathbf{n} \text {. }
$$

Based on (10) we construct the proposed beamformer $\mathbf{P}$ as

$$
\mathbf{P}=\frac{1}{f} \cdot \mathbf{Z}^{-1} \mathbf{W}
$$

where following the formulation of closed-form beamformers $f=\left\|\mathbf{Z}^{-1} \mathbf{W}\right\|$ is the scaling factor to ensure that the signal power is not changed after beamforming, and (10) is further transformed into

$$
\mathbf{y}=\frac{1}{f} \cdot \mathbf{D W} \mathbf{s}+\mathbf{n} .
$$

As can be observed from (12), with this beamforming structure the mutual coupling effect can be eliminated in the channel, while it still has an effect on the system performance, which is fully characterized by the resulting scaling factor $f$. Moreover, the proposed beamforming structure further simplifies the joint beamforming design, as shown in the following. With the proposed beamformer, the received SINR for the $k$ th user in (3) is then transformed into

$$
\gamma_{k}=\frac{\left|\mathbf{d}_{k} \mathbf{w}_{k}\right|^{2}}{\sum_{i \neq k}\left|\mathbf{d}_{k} \mathbf{w}_{i}\right|^{2}+f^{2} \sigma^{2}},
$$

where $\mathbf{W}=\left[\mathbf{w}_{1}, \mathbf{w}_{2}, \ldots, \mathbf{w}_{K}\right]$ and $f$ can also be seen as a noise amplification factor. In (13), it is observed that the received SINR $\gamma_{k}$ is related to both $\mathbf{w}_{k}$ and $f$, and therefore by jointly optimizing $\mathbf{W}$ and selecting the impedance value for each load, we can maximize the minimum received SINR. We then formulate the optimization problem as

$$
\begin{array}{ll}
\mathcal{P}_{0}: & \max _{\mathbf{z}_{L}, \mathbf{W}} \min _{k} \gamma_{k} \\
\text { s.t. } & \Re\left(z_{L_{i}}\right) \geq 0, \forall i \in \mathcal{I}
\end{array}
$$

where $\mathcal{I}=\left\{1,2, \ldots, N_{t}\right\}$. We note that unlike conventional SINR balancing problem that contains a power budget in the optimization constraints, our formulation of the optimization $\mathcal{P}_{0}$ does not have such an explicit power constraint. This is due to the introduction of the scaling factor $f$ for the constructed beamformer in (11), which indeed ensures that the transmit power is constrained. The constraint on the value of each $z_{L_{i}}$ is from the fact that in practice the real part of the load impedances should be positive such that the antenna array can radiate power [50][51]. Due to the fact that $f=\left\|\mathbf{Z}^{-1} \mathbf{W}\right\|$, the optimization problem in (14) is not jointly convex in $\mathbf{z}_{L}$ and $\mathbf{W}$, and cannot be directly solved by convex optimization. Nevertheless, we note that $\mathcal{P}_{0}$ can be transformed into a biconvex problem, i.e., it is convex with respect to $\mathbf{W}$ when $\mathbf{z}_{L}$ is fixed and vice versa. Therefore, in the following we propose to optimize $\mathbf{W}$ and $\mathbf{z}_{L}$ alternately.

\section{A. Solving $\mathbf{W}$ when $\mathbf{z}_{L}$ is fixed}

When $\mathbf{z}_{L}$ is fixed, the optimization problem $\mathcal{P}_{0}$ is reduced to a digital beamforming problem, which can be expressed as

$$
\begin{array}{ll}
\mathcal{P}_{1}: & \max _{\mathbf{W}} \min _{k} \gamma_{k} \\
\text { s.t. } & \left\|\mathbf{Z}^{-1} \mathbf{W}\right\|^{2}=M
\end{array}
$$

where we note that the power constraint is only a temporary constraint for the SINR balancing sub-problem and indeed in $\mathcal{P}_{1} M$ can be any arbitrary positive real value. This will not have an impact on the solution of the original optimization problem $\mathcal{P}_{0}$ because of the scaling factor $f$ that constrains the power of the beamformer. It can be observed that, due to the strict equality constraint, $\mathcal{P}_{1}$ is not convex. To solve $\mathcal{P}_{1}$, we firstly consider the following SINR balancing beamforming, which is expressed as

$$
\begin{array}{ll}
\mathcal{P}_{2}: & \max _{\mathbf{W}} \min _{k} \gamma_{k} \\
\text { s.t. } & \left\|\mathbf{Z}^{-1} \mathbf{W}\right\|^{2} \leq M
\end{array}
$$

where we optimize $\mathbf{W}$ while $\mathbf{Z}$ is fixed. Based on the SINR expression and [16][17], it is easily observed that the power constraint for $\mathcal{P}_{2}$ is strictly active when optimality is achieved, which means that $\mathcal{P}_{2}$ is indeed equivalent to $\mathcal{P}_{1}$ and the optimal solution to $\mathcal{P}_{2}$ is also optimal for $\mathcal{P}_{1}$. Therefore, in the following we propose to solve $\mathcal{P}_{2}$ instead. Compared to conventional SINR balancing problem in [16][17], the SINR optimization problem here differs in that the power constraint is on $\mathbf{Z}^{-1} \mathbf{W}$ instead of $\mathbf{W}$. Thanks to the fact that $\mathbf{Z}^{-1}$ is regarded as fixed when optimizing $\mathbf{W}$, we can apply existing algorithms that solve conventional SINR balancing problem 
to solve $\mathcal{P}_{2}$ with $\mathbf{z}_{L}$ fixed by either the iterative scheme in [16] or the bisection search in [17], and we discuss the initialization of $\mathbf{z}_{L}$ in Section III-C below. In this paper, we employ the bisection search scheme to solve $\mathcal{P}_{2}$ based on the fact that SINR balancing and power minimization problems are inverse problems [17]. To be specific, we firstly express the power minimization problem that corresponds to the SINR balancing problem $\mathcal{P}_{2}$ as a second-order-cone-programming (SOCP) problem, given by

$$
\begin{array}{ll}
\mathcal{P}_{3}: & \min _{\mathbf{W}} p_{0} \\
\text { s.t. } & A_{k}(\mathbf{W}) \leq 0, \forall k \in \mathcal{K} \\
& C(\mathbf{W}) \leq 0
\end{array}
$$

where $\mathcal{K}=\{1,2, \cdots, K\}$ and $p_{0}$ is the power variable to be optimized for the corresponding power minimization problem. $A_{k}(\mathbf{W})$ is given by

$A_{k}(\mathbf{W})=\left\|\mathbf{W}^{H} \mathbf{D}^{H} \mathbf{I}_{k}\right\|+\sigma-\sqrt{1+\frac{1}{\gamma_{0}}} \cdot[\mathbf{D W}]_{k, k}, \forall k \in \mathcal{K}$,

where we have assumed that $[\mathbf{D W}]_{k, k}>0 . C(\mathbf{W})$ is given by

$$
C(\mathbf{W})=\left\|\mathbf{Z}^{-1} \mathbf{W}\right\|-p_{0} .
$$

In (18), $\gamma_{0}$ is the SINR target for the power minimization problem $\mathcal{P}_{3}$ and we further denote the power minimization problem as $\mathcal{P}_{3}\left(\gamma_{0}\right) . \mathcal{P}_{3}$ is an SOCP problem and can be efficiently solved by standard SOCP solvers or interior point methods.

As the SINR balancing optimization $\mathcal{P}_{2}$ and power optimization problem $\mathcal{P}_{3}\left(\gamma_{0}\right)$ are inverse problems, we can solve $\mathcal{P}_{2}$ by iteratively solving the corresponding power optimization problems for different SINR requirement $\gamma_{0}$. Due to the inversion property, if $\mathcal{P}_{3}\left(\gamma_{0}\right)=M$, then its solution will be optimal for $\mathcal{P}_{2}$ [17]. We then present the following algorithm to efficiently solve $\mathcal{P}_{2}$, summarized below in Algorithm 1, where $\gamma_{\min }$ and $\gamma_{\max }$ define the range of SINRs.

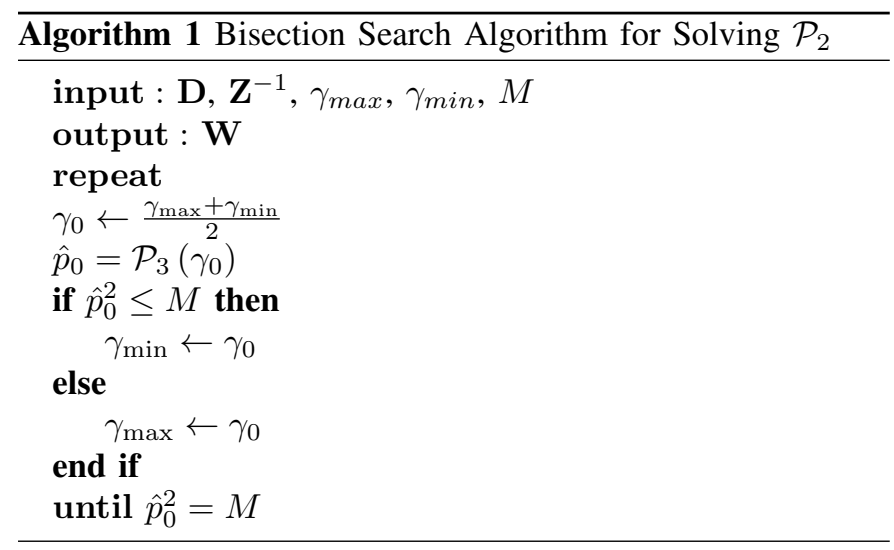

\section{B. Solving $\mathbf{z}_{L}$ when $\mathbf{W}$ is fixed}

When $\mathbf{W}$ is fixed, $\mathcal{P}_{0}$ is transformed into

$$
\begin{array}{ll}
\mathcal{P}_{4}: & \max _{\mathbf{z}_{L}} \min _{k} \gamma_{k} \\
\text { s.t. } & \Re\left(z_{L_{i}}\right) \geq 0, \forall i \in \mathcal{I} \\
& f=\left\|\mathbf{Z}^{-1} \mathbf{W}\right\|
\end{array}
$$

Then, based on the expression of $\gamma_{k}$ in (13), it is observed that the noise amplification factor $f$ is the only variable when $\mathbf{W}$ is fixed. Therefore, $\mathcal{P}_{4}$ is equivalent to minimizing the noise amplification factor $f$ and can be further transformed into

$$
\begin{array}{ll}
\mathcal{P}_{5}: & \min _{\mathbf{z}_{L}}\left\|\mathbf{Z}^{-1} \mathbf{W}\right\| \\
\text { s.t. } & \Re\left(z_{L_{i}}\right) \geq 0, \forall i \in \mathcal{I}
\end{array}
$$

where we optimize each load value $z_{L_{i}}$ to minimize $f$. To solve (21), we firstly study the inverse of the mutual coupling matrix $\mathbf{Z}^{-1}$. Based on (7), $\mathbf{Z}^{-1}$ is obtained as

$$
\begin{aligned}
\mathbf{Z}^{-1} & =\left\{\left[z_{A} \cdot \mathbf{I}+\operatorname{diag}\left(\mathbf{z}_{L}\right)\right]\left[\boldsymbol{\Gamma}+\operatorname{diag}\left(\mathbf{z}_{L}\right)\right]^{-1}\right\}^{-1} \\
& =\left[\boldsymbol{\Gamma}+\operatorname{diag}\left(\mathbf{z}_{L}\right)\right] \cdot \operatorname{diag}\left(\mathbf{z}_{T}\right)
\end{aligned}
$$

where

$$
\mathbf{z}_{T}=\left[\frac{1}{z_{1}}, \frac{1}{z_{2}}, \ldots, \frac{1}{z_{N_{t}}}\right]^{T}, z_{i}=z_{A}+z_{L_{i}} .
$$

By expanding (22), we have

$$
\mathbf{Z}^{-1}=\left[\begin{array}{ccccc}
1 & \frac{z_{m_{1}}}{z_{2}} & \frac{z_{m_{2}}}{z_{3}} & \cdots & \frac{z_{m_{N_{t}-1}}}{z_{N_{t}}} \\
\frac{z_{m_{1}}}{z_{1}} & 1 & \frac{z_{m_{1}}}{z_{3}} & \ddots & \vdots \\
\frac{z_{m_{2}}}{z_{1}} & \frac{z_{m_{1}}}{z_{2}} & \ddots & \ddots & \frac{z_{m_{2}}}{z_{N_{t}}} \\
\vdots & \ddots & \ddots & \ddots & \frac{z_{m_{1}}}{z_{N_{t}}} \\
\frac{z_{m_{N_{t}-1}}}{z_{1}} & \cdots & \frac{z_{m_{2}}}{z_{N_{t}-2}} & \frac{z_{m_{1}}}{z_{N_{t}-1}} & 1
\end{array}\right] .
$$

Then, by denoting

$$
\frac{z_{m_{1}}}{z_{i}}=\theta_{i}
$$

$\mathbf{Z}^{-1}$ in (22) can be further decomposed into

$$
\mathbf{Z}^{-1}=\mathbf{B} \Theta+\mathbf{I}
$$

where $\boldsymbol{\Theta}=\operatorname{diag}\left(\theta_{1}, \theta_{2}, \ldots, \theta_{N_{t}}\right)$ and the matrix $\mathbf{B}$ is given as

$$
\mathbf{B}=\left[\begin{array}{ccccc}
0 & 1 & \frac{z_{m_{2}}}{z_{m_{1}}} & \cdots & \frac{z_{m_{N_{t}-1}}}{z_{m_{1}}} \\
1 & 0 & 1 & \ddots & \vdots \\
\frac{z_{m_{2}}}{z_{m_{1}}} & 1 & \ddots & \ddots & \frac{z_{m_{2}}}{z_{m_{1}}} \\
\vdots & \ddots & \ddots & \ddots & 1 \\
\frac{z_{m_{N_{t}-1}}}{z_{m_{1}}} & \cdots & \frac{z_{m_{2}}}{z_{m_{1}}} & 1 & 0
\end{array}\right] .
$$

As for the constraint $\Re\left(z_{L_{i}}\right) \geq 0$, based on (23) and (25) we can express the load impedance $z_{L_{i}}$ as

$$
z_{L_{i}}=\frac{z_{m_{1}}}{\theta_{i}}-z_{A}
$$

Then based on (28), the constraint in $\mathcal{P}_{5}$ can be further transformed into

$$
\begin{aligned}
& \Re\left(\frac{z_{m_{1}}}{\theta_{i}}\right) \geq \Re\left(z_{A}\right) \\
\Rightarrow & \frac{\Re\left(\theta_{i}\right) \Re\left(z_{m_{1}}\right)+\Im\left(\theta_{i}\right) \Im\left(z_{m_{1}}\right)}{\left\|\theta_{i}\right\|^{2}} \geq \Re\left(z_{A}\right) \\
\Rightarrow & \Re\left(\theta_{i}\right) \Re\left(z_{m_{1}}\right)+\Im\left(\theta_{i}\right) \Im\left(z_{m_{1}}\right) \geq\left\|\theta_{i}\right\|^{2} \Re\left(z_{A}\right)
\end{aligned}
$$


which is also convex, where we note that a practical antenna has $\Re\left(z_{A}\right)>0$ [33]. Therefore, by substituting (26) and (29) into $\mathcal{P}_{5}$, the optimization problem is finally formulated as

$$
\begin{array}{ll}
\mathcal{P}_{6}: & \min _{\Theta}\|\mathbf{B} \boldsymbol{\Theta} \mathbf{W}+\mathbf{W}\|^{2} \\
\text { s.t. } & \Re\left(\theta_{i}\right) \Re\left(z_{m_{1}}\right)+\Im\left(\theta_{i}\right) \Im\left(z_{m_{1}}\right) \geq\left\|\theta_{i}\right\|^{2} \Re\left(z_{A}\right), \forall i \in \mathcal{I}
\end{array}
$$

which is a least-squares problem and can be efficiently solved with convex optimization tools. Then, each $z_{L_{i}}$ can be obtained by (28) and the resulting optimal mutual coupling matrix is obtained as

$$
\mathbf{Z}^{*}=\left(\mathbf{B} \Theta^{*}+\mathbf{I}\right)^{-1}
$$

\section{Joint Iterative Algorithm}

Based on the above, the proposed joint analog-digital beamformer can be obtained by alternately optimzing $\mathbf{W}$ and $\mathbf{z}_{L}$ until convergence or a maximum number of iterations is reached. We then summarize the algorithm for the joint scheme in Algorithm 2, where $\mathbf{z}_{L}^{0}$ is the initial load impedance vector, and $N_{\max }$ is the maximum iteration number. $\delta$ is a variable that represents the convergence accuracy, which is defined as $\delta=\left\|\mathbf{W}^{(n+1)}-\mathbf{W}^{(n)}\right\|$ (or equivalently $\delta=$ $\left\|\mathbf{Z}^{(n+1)}-\mathbf{Z}^{(n)}\right\|$ ). $\delta_{t h}$ defines the accuracy threshold.

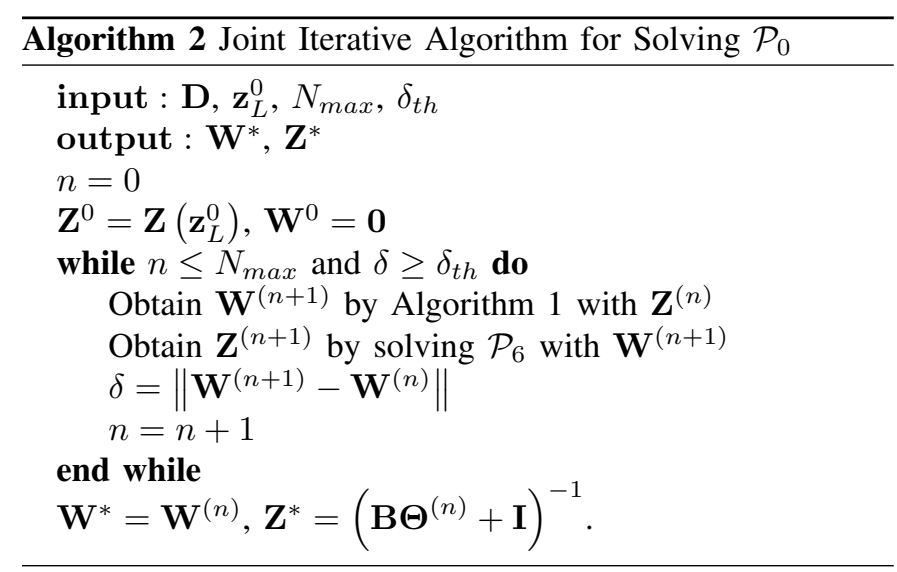

Convergence: It is observed in Algorithm 2 that for each iteration the two sub-problems have the same objective function $\mathcal{P}_{0}$ and are transformed into equivalent problems $\mathcal{P}_{2}$ and $\mathcal{P}_{4}$. Moreover, the sub-problem $\mathcal{P}_{2}$ to obtain $\mathbf{W}^{(n)}$ and the sub-problem $\mathcal{P}_{4}$ to obtain $\mathbf{Z}^{(n)}$ are optimally solved by Algorithm 1 and $\mathcal{P}_{6}$ for that iteration, respectively. Therefore, Algorithm 2 is guaranteed to converge [52][53], and it is easy to observe that when the global optimum is reached, $f^{*}=M$. More importantly, it is found that the convergence speed of the proposed joint algorithm is fast, which is shown below in Fig. 2, where we plot the value of both $f$ and $\delta$ with respect to the iteration number $n$ and for simplicity we assume that $M=1$. 'SB A-D Joint' denotes the proposed joint AD SINR balancing optimization. In Fig. 2, the initial load impedance vector $\mathbf{z}_{L}^{0}$ is $z_{L_{i}}=50 \Omega, \forall i \in \mathcal{I}, \gamma_{\min }=0$, and $\gamma_{\max }$ is selected as $\gamma_{\max }=\frac{\max \left(\left\|\mathbf{h}_{k}\right\|^{2}\right)}{\sigma^{2}}$. It can be observed that the proposed algorithm is convergent within $n=4$ iterations.

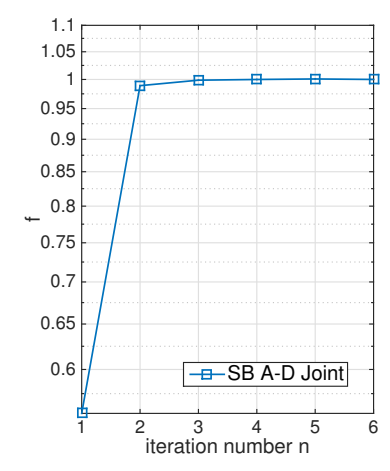

(a) Value of $f$

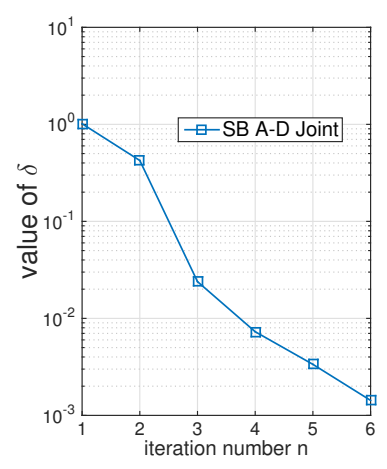

(b) Value of $\delta$
Fig. 2: Convergence speed of the joint iterative scheme, $N_{t}=$ $K=4, N_{\max }=6, \delta_{t h}=10^{-2}$

\section{Low-Complexity AD BeAmForming AND DeALING WITH QUANTIZED ANALOG LOADS}

In this section, based on the proposed joint beamforming scheme in Section III, we further propose two practical beamforming schemes to exploit the mutual coupling effect. First we explore a low-complexity alternative to the joint optimization above. We then focus on the practical case where only quantized versions of the analog loads are available, and present a practical scheme for the analog beamforming optimization.

\section{A. Decoupled Low-Complexity Beamforming Scheme}

Due to the fact that $\mathbf{Z}^{-1}$ and $\mathbf{W}$ need to be updated alternately, the proposed joint scheme above involves considerable computational complexity. Therefore in this section, a more practical decoupled low-complexity scheme is introduced, where the digital beamformer is firstly obtained, followed by the optimization on each load impedance. Furthermore, we note that in practice closed-form beamforming schemes such as ZF and regularized (RZF) are widely applied due to their complexity benefits. While the joint AD beamforming scheme in Section III does not directly apply to closed-form beamforming approaches, the decoupled scheme in this section enables the combination of mutual coupling exploitation with such closed-form schemes, which leads to much reduced computational complexity.

For the proposed decoupled scheme, we first construct the proposed beamformer $\mathbf{P}$ as in (11), and we further denote $\mathbf{W}_{d}$ as the digital beamformer for the decoupled scheme. $\mathbf{W}_{d}$ is firstly obtained with conventional digital schemes, and we then design $\mathbf{z}_{L}$ to minimize the noise amplification factor $f$. The decoupled beamforming scheme is then summarized in Algorithm 3 below.

With the proposed decoupled approach, the computational complexity is greatly reduced, while it will be observed that for SINR balancing beamforming there is only a slight performance loss compared to the joint approach. 


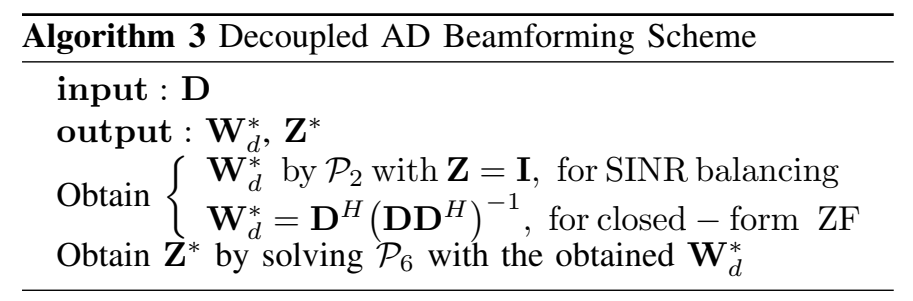

\section{B. Sequential Search Scheme for the Optimization with Quan- tized Loads based on Greedy Algorithm}

While in the above sections we have considered the realistic constraint for the varactors where $\Re\left(z_{L_{i}}\right) \geq 0, \forall i \in \mathcal{I}$, this constraint is from the perspective of enabling the radiation of the antenna array. In the above optimization problems, it can be observed that we have assumed that each $z_{L_{i}}$ can have any arbitrary continuous values, which means that infinite precision of the varactors is assumed. This assumption may not be feasible, since hardware components with finite precision are used in the practical implementation. Therefore, in this section we consider the mutual coupling exploitation with quantized tunable loads. In this case, the constraint for the tunable load is modified into

$$
z_{L_{i}} \in \mathcal{S}, \forall i \in \mathcal{I}
$$

where $\mathcal{S}$ is the set of quantized load values with a finite number. For the quantization-based beamforming scheme, the optimization problem $\mathcal{P}_{5}$ in (21) for $\mathbf{z}_{L}$ can be reformulated as

$$
\begin{array}{ll}
\mathcal{P}_{7}: & \min _{\mathbf{z}_{L}}\left\|\mathbf{Z}^{-1} \mathbf{W}\right\| \\
\text { s.t. } & z_{L_{i}} \in \mathcal{S}, \forall i \in \mathcal{I}
\end{array}
$$

We denote each potential load value in $\mathcal{S}$ as $z_{k}^{\text {cand }}$, and we further denote $D_{R}$ and $D_{I}$ as the total number of potential values for the real and imaginary part of each $z_{k}^{\text {cand }} \in \mathcal{S}$ respectively. Then, the cardinality of $\mathcal{S}$ can be obtained as

$$
\operatorname{card}(\mathcal{S})=D_{R} D_{I}=D .
$$

Then, each $z_{k}^{\text {cand }} \in \mathcal{S}$ can be further expressed as

$$
\begin{aligned}
& \Re\left(z_{k}^{\text {cand }}\right) \in\left\{z_{1}^{R E}, z_{2}^{R E}, \ldots, z_{D_{R}}^{R E}\right\}, \\
& \Im\left(z_{k}^{\text {cand }}\right) \in\left\{z_{1}^{I M}, z_{2}^{I M}, \ldots, z_{D_{I}}^{I M}\right\},
\end{aligned}
$$

where the quantized potential load values $z_{p}^{R E}$ and $z_{q}^{I M}$ in (35) can be obtained as

$$
\begin{aligned}
& z_{p}^{R E}=\frac{p \cdot R_{\text {upper }}}{D_{R}}, \forall p \in\left\{1,2, \ldots, D_{R}\right\} \\
& z_{q}^{I M}=I_{\text {lower }}+\frac{q \cdot\left(I_{\text {upper }}-I_{\text {lower }}\right)}{D_{I}}, \forall q \in\left\{1,2, \ldots, D_{I}\right\} .
\end{aligned}
$$

In (36), $R_{\text {upper }}$ denotes the upper bound for the real part of each $z_{k}^{\text {cand }} \in \mathcal{S}$ where we note that the lower bound for the real part is 0 as seen in (14), while $I_{\text {lower }}$ and $I_{\text {upper }}$ denote the lower bound and upper bound for the imaginary part of $z_{k}^{\text {cand }} \in \mathcal{S}$. We note that here we also constrain the imaginary part of each $z_{L_{i}}$ in a finite range for practical consideration.

It is then observed that to obtain the optimal quantized load vector, an exhaustive search over a total number of $D$ possible load values is required for each antenna, which is highly computationally expensive, especially when the number of antennas $N_{t}$ is large or the cardinality of $\mathcal{S}$ is large. Therefore, in this section we propose a low-complexity sequential search scheme based on a greedy algorithm, where for the load impedance of each antenna element, we sequentially select the load value that provides the highest performance improvements, which is achieved by selecting the load value that generates the lowest value of $f$, while assuming the loads on other antenna elements are fixed. The proposed scheme is then summarized in Algorithm 4, which can be applied upon the joint scheme in Section III-C or the decoupled scheme in Section IV-A to efficiently obtain the load value for each antenna element, when the considered tunable loads are quantized and the exhaustive search scheme is too complicated to be implemented.

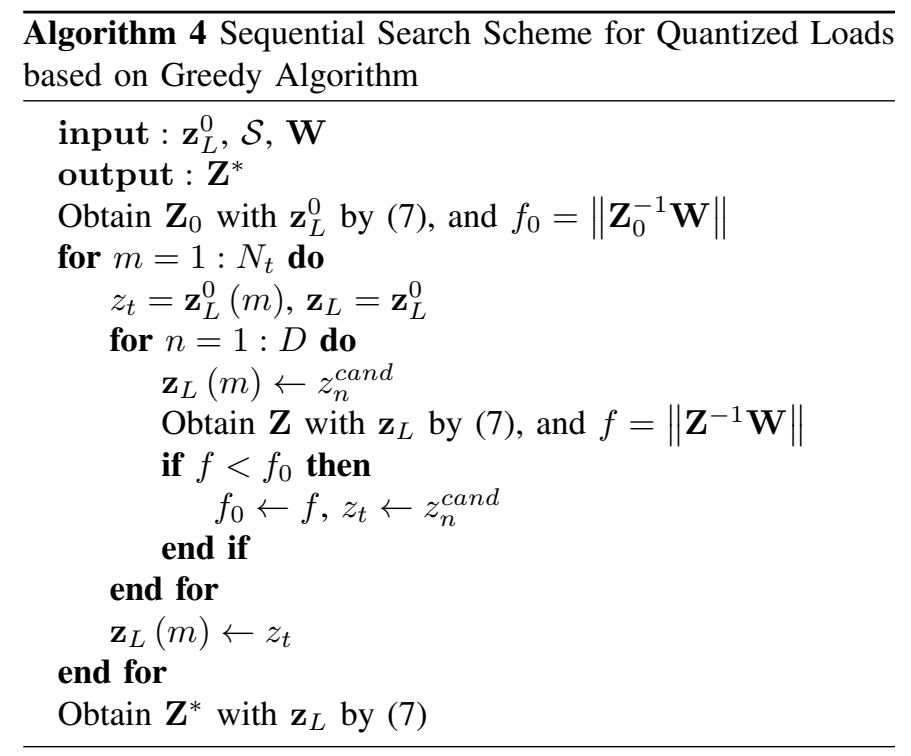

Furthermore, to numerically show the complexity benefits of the proposed scheme, we firstly express the total number of possible load combinations to visit for both the exhaustive search scheme and the proposed sequential search scheme, obtained as

$$
\begin{aligned}
& N_{\text {exhaustive }}=D^{N_{t}}=\left(D_{R} D_{I}\right)^{N_{t}}, \\
& N_{\text {sequential }}=D N_{t}=D_{R} D_{I} N_{t} .
\end{aligned}
$$

An illustrative example is then presented in Table I below with some typical values of the antenna number and quantization level, and we can observe a significant complexity gain for the proposed sequential search scheme, compared to the exhaustive search scheme which is of high complexity.

\section{Estimation of D AND the Imperfect CSI Model}

\section{A. Estimation of $\mathbf{D}$}

We observe that the BS needs to estimate $\mathbf{D}$ to perform the proposed schemes. In this paper, we assume CSI can be obtained by the BS with channel estimation techniques based on the duplex mode. Nevertheless, with channel estimation schemes we can only obtain $\mathbf{H}=\mathbf{D Z}$. Therefore, firstly we need to extract $\mathbf{D}$ from $\mathbf{D Z}$ to facilitate the proposed schemes. We note that the mutual impedance matrix $\boldsymbol{\Gamma}$ is 


\begin{tabular}{|c|c|c|c|c|c|}
\hline \multicolumn{7}{|c|}{ Exhaustive Search Scheme } \\
\hline \multirow{2}{*}{ Antenna Number } & \multicolumn{5}{|c|}{ Quantization Level } \\
\cline { 2 - 6 } & 2 & 4 & 6 & 8 & 10 \\
\hline 4 & 256 & $6.6 \times 10^{4}$ & $1.7 \times 10^{6}$ & $1.7 \times 10$ & $1 \times 10^{8}$ \\
\hline 8 & $6.6 \times 10^{4}$ & $4.3 \times 10^{9}$ & $2.8 \times 10^{12}$ & $2.8 \times 10^{14}$ & $1 \times 10^{16}$ \\
\hline 16 & $4.3 \times 10^{9}$ & $1.8 \times 10^{19}$ & $8 \times 10^{24}$ & $7.9 \times 10^{28}$ & $1 \times 10^{32}$ \\
\hline \multicolumn{7}{|c|}{ Sequential Search Scheme } \\
\hline \multirow{2}{*}{ Antenna Number } & 2 & 4 & 6 & 8 & 10 \\
\cline { 2 - 6 } & 16 & 64 & 144 & 256 & 400 \\
\hline 4 & 32 & 128 & 288 & 512 & 800 \\
\hline 8 & 64 & 256 & 576 & 1024 & 1600 \\
\hline 16 & \multicolumn{7}{|c|}{ Quantization Level } \\
\hline
\end{tabular}

TABLE I: Number of load combinations to visit for the exhaustive search and proposed sequential search scheme

only dependent on the array structure and does not change with varying channel states, and further $\boldsymbol{\Gamma}$ is typically known to the BS either by the induced EMF method or other experimental measurements such as boundary-value approach and transmission-line method [33]. Before data transmission, we can firstly set each load impedance to a specific reference value, for example $z_{L_{i}}=50 \Omega, \forall i \in \mathcal{I}$, and we denote the resulting load impedance vector as $\mathbf{z}_{L}^{0}$ and the mutual coupling matrix as $\mathbf{Z}_{0}$. Then, the BS obtains the estimate of the channel $\mathbf{H}$ with $\mathbf{z}_{L}^{0}$. With $\mathbf{z}_{L}^{0}$ and further $\mathbf{Z}_{0}$ known to the BS, $\mathbf{D}$ can be extracted from $\mathbf{D Z} \mathbf{Z}_{0}$ and expressed as

$$
\mathbf{D}=\mathbf{H Z}_{0}^{-1} .
$$

Then, the proposed schemes can be applied during data transmission.

\section{B. Imperfect CSI Model}

In practical wireless communication systems, there exist errors in obtaining the CSI. In this paper, we consider two typical CSI error models, which correspond to the time division duplex (TDD) mode and the frequency division duplex (FDD) mode, respectively.

1) Statistical CSI Error Model: For the TDD mode, the CSI can be directly obtained at the transmitter using reciprocity and is subject to noise errors, as discussed in [53] and the references therein. For this case, the MMSE channel estimation is employed with an orthogonal training method as described in [54], and we assume the channel remains constant within a time slot. Then, the imperfect channel model with statistical CSI errors can be obtained as

$$
\mathbf{H}=\alpha \cdot(\hat{\mathbf{H}}+\mathbf{E})+\mathbf{Q},
$$

where $\mathbf{H}=\mathbf{D Z}$ represents the channel at transmission and $\hat{\mathbf{H}}=\hat{\mathbf{D}} \mathbf{Z}$ is the estimated channel. In (39), $\alpha$ is known as a correlation factor and $\mathbf{Q}$ is a delay error matrix, where the elements in $\mathbf{Q}$ are i.i.d. random variables that follow $\mathcal{C N}\left(0,1-\alpha^{2}\right)$. $\mathbf{E}$ is the estimation error matrix with each element $e_{i, j} \sim \mathcal{C N}(0, \eta), \forall i \in\{1,2, \ldots, K\}, \forall j \in\left\{1,2, \ldots, N_{t}\right\}$, and $\eta$ is given by

$$
\eta=\beta \cdot\left(\frac{1}{\sigma^{2}}\right)^{-1},
$$

where $\beta$ is the inverse proportionality coefficient, with $\rho=\frac{1}{\sigma^{2}}$ being the transmit SNR. In (39) and (40), $\alpha=1$ and $\beta=0$ models the perfect CSI.

Based on the previous discussion we need to extract $\mathbf{D}$ from $\mathbf{H}$ to perform the proposed schemes, and then with (38) and the imperfect CSI model in (39) we can express $\mathbf{D}$ as

$$
\begin{aligned}
\mathbf{D} & =\alpha \cdot\left(\hat{\mathbf{D}}+\mathbf{E} \mathbf{Z}^{-1}\right)+\mathbf{Q Z}^{-1} \\
& =\alpha \cdot \hat{\mathbf{D}}+(\alpha \cdot \mathbf{E}+\mathbf{Q}) \mathbf{Z}^{-1} .
\end{aligned}
$$

2) Norm Bounded CSI Error Model: When the system is operating in FDD mode, the norm bounded CSI error model is prevalently considered to model the inaccuracy caused by quantization in limited feedback [55][56]. Then, after the estimation of $\mathbf{D}$ with the approach introduced in Section V-A, the norm bounded CSI error model can be expressed as: for each user $k$,

$$
\mathbf{d}_{k}=\hat{\mathbf{d}}_{k}+\mathbf{e}_{k}, \forall k \in\{1,2, \ldots, K\} .
$$

Similar to [55][56], the channel uncertainty can be considered as bounded by a spherical region, expressed as

$$
\mathcal{D}_{k}:=\left\{\hat{\mathbf{d}}_{k}+\mathbf{e}_{k} \mid\left\|\mathbf{e}_{k}\right\| \leq \sqrt{\delta_{k}}\right\}, \forall k \in\{1,2, \ldots, K\},
$$

where $\delta_{k}$ is the channel inaccuracy coefficient that defines the radius of $\mathcal{D}_{k}$.

\section{Vi. Performance Metrics and the Optimality for CLOSED-FORM BEAMFORMERS}

In this section, the received SINR and resulting sum rate are firstly shown under perfect CSI. Subsequently, for the case of imperfect CSI, by calculating the SINR of the received symbols we show that for closed-form beamforming techniques, the load values obtained by perfect CSI are also optimal.

\section{A. Perfect CSI}

With perfect CSI, the received SINR of user $k$ for SINR balancing can be obtained by (13), based on which the achievable sum rate can be obtained as

$$
R=\sum_{k=1}^{K} \log _{2}\left(1+\gamma_{k}\right) .
$$

We then proceed to calculate the received SINR for imperfect CSI, where we employ the statistical CSI error model as an example, and the extension to the norm bounded CSI can be similarly obtained.

\section{B. Imperfect CSI - Statistical CSI Errors}

Based on (1)(41), when the proposed scheme is applied with the statistical CSI errors, the received signal vector $\hat{\mathbf{y}}$ can be expressed as

$$
\begin{aligned}
\hat{\mathbf{y}} & =\mathbf{H} \hat{\mathbf{x}}+\mathbf{n} \\
& =\left[\alpha \cdot \hat{\mathbf{D}}+(\alpha \cdot \mathbf{E}+\mathbf{Q}) \mathbf{Z}^{-1}\right] \mathbf{Z} \cdot \frac{1}{\hat{f}} \mathbf{Z}^{-1} \hat{\mathbf{W}} \mathbf{s}+\mathbf{n} \\
& =\frac{\alpha}{\hat{f}} \cdot \hat{\mathbf{D}} \hat{\mathbf{W}} \mathbf{s}+\frac{1}{\hat{f}} \cdot(\alpha \cdot \mathbf{E}+\mathbf{Q}) \mathbf{Z}^{-1} \hat{\mathbf{W}} \mathbf{s}+\mathbf{n},
\end{aligned}
$$


where $\hat{\mathbf{W}}$ can denote $\hat{\mathbf{W}}^{*}$ or $\hat{\mathbf{W}}_{d}^{*}$. It is observed from (45) that in the presence of statistical CSI errors, each receiver needs to rescale the received signal by $\frac{\hat{f}}{\alpha}$, and the resulting received symbol vector can be expressed as

$$
\hat{\mathbf{r}}=\hat{\mathbf{D}} \hat{\mathbf{W}} \mathbf{s}+\left(\mathbf{E}+\frac{1}{\alpha} \cdot \mathbf{Q}\right) \mathbf{Z}^{-1} \hat{\mathbf{W}} \mathbf{s}+\frac{\hat{f}}{\alpha} \cdot \mathbf{n} .
$$

The second term in (46) acts as an additional noise that is introduced by the imperfect CSI. We then define the equivalent noise term as

$$
\hat{\mathbf{n}}=\left(\mathbf{E}+\frac{1}{\alpha} \cdot \mathbf{Q}\right) \mathbf{Z}^{-1} \hat{\mathbf{W}} \mathbf{s}+\frac{\hat{f}}{\alpha} \cdot \mathbf{n} .
$$

Based on the derivation in [57], it is shown that the distribution of $\hat{\mathbf{n}}$ conditioned on $\hat{\mathbf{D}}$ and $\mathbf{s}$ is i.i.d. Gaussian with zero mean, i.e. $\hat{\mathbf{n}} \sim \mathcal{C N}(0, v \cdot \mathbf{I})$, with the equivalent noise power given by

$$
\begin{aligned}
v & =\left[\eta+\frac{\left(1-\alpha^{2}\right)}{\alpha^{2}}\right] \cdot \hat{f}^{2}+\frac{\hat{f}^{2} \sigma^{2}}{\alpha^{2}} \\
& =\frac{\hat{f}^{2}}{\alpha^{2}}\left[\alpha^{2} \eta+\left(1-\alpha^{2}\right)+\sigma^{2}\right]
\end{aligned}
$$

where we note that $\hat{\mathbf{x}}=\frac{1}{\hat{f}} \cdot \mathbf{Z}^{-1} \hat{\mathbf{W}} \mathbf{s}$ is normalized, and therefore we have $\|\hat{\mathbf{x}}\|^{2}=1$ and further $\left\|\mathbf{Z}^{-1} \hat{\mathbf{W}} \mathbf{s}\right\|^{2}=\hat{f}^{2}$. It is observed that the derivation in (45)-(48) is independent of the beamforming schemes applied in the digital domain. Then, we can express the resulting received SINR for the $k$-th user with statistical CSI errors as

$$
\hat{\gamma}_{k}=\frac{\left|\hat{\mathbf{d}}_{k} \hat{\mathbf{w}}_{k}^{*}\right|^{2}}{\sum_{i \neq k}\left|\hat{\mathbf{d}}_{k} \hat{\mathbf{w}}_{i}^{*}\right|^{2}+\frac{\hat{f}^{2}}{\alpha^{2}}\left[\alpha^{2} \eta+\left(1-\alpha^{2}\right)+\sigma^{2}\right]},
$$

and the achievable sum rate can be obtained by (44) based on $\hat{\gamma}_{k}$. Based on the observation in (49), we note the optimality of the proposed $\mathrm{AD}$ schemes for closed-form beamforming schemes with imperfect CSI, where in such case, each $\hat{\mathrm{w}}_{i}^{*}$, $\forall i \in\{1,2, \ldots, K\}$ in (49) is only dependent on the CSI and is fixed. Then, the received SINR of the $k$-th user with statistical CSI errors is only relevant to the noise amplification factor $\hat{f}$. Therefore for the proposed analog beamforming schemes that minimize the noise amplification factor $\hat{f}$, the power of the equivalent noise vector $\hat{\mathbf{n}}$ for the imperfect CSI scenarios is also minimized, which means that the solutions obtained with perfect CSI are also optimal for the case of statistical CSI errors, and the proposed schemes can be directly extended to such cases.

\section{Imperfect CSI - Norm Bounded CSI Errors}

When the CSI errors are norm bounded, the optimality trivially applies to the case where closed-form beamformers are employed in the digital domain. In such case, instead of obtaining the exact analytical expression, we can obtain the lower bound of the received SINR based on a similar derivation in (45)-(48), expressed as

$$
\hat{\gamma}_{k} \geq \frac{\left|\hat{\mathbf{d}}_{k} \hat{\mathbf{w}}_{k}^{*}\right|^{2}}{\sum_{i \neq k}\left|\hat{\mathbf{d}}_{k} \hat{\mathbf{w}}_{i}^{*}\right|^{2}+\hat{f}^{2} \sigma^{2}+\delta_{k}}=\hat{\gamma}_{k}^{\text {lower }} .
$$

\section{RobUSt DESIGN FOR SINR OptIMIZATION}

In this section and based on the previous equivalent noise power analysis, the robust design for SINR optimization with imperfect CSI is proposed, where we propose the robust scheme for the statistical CSI error model and the norm bounded CSI error, respectively.

\section{A. Robust Design - Statistical CSI Errors}

Based on the derivation in (45)-(48), the received SINR with statistical CSI errors can be regarded similar to the perfect CSI case with an equivalent noise term $\hat{\mathbf{n}}$. It is further noted that the power of $\hat{\mathbf{n}}$ is independent of the mutual coupling matrix $\mathbf{Z}$. Therefore, for the robust SINR balancing optimization with statistical CSI errors, instead of applying the noise power of $\mathbf{n}$, we apply the power of the equivalent noise $\hat{\mathbf{n}}$ for $A_{k}(\mathbf{W})$ in (18), and we then obtain $\hat{A}_{k}(\mathbf{W})$ as

$$
\hat{A}_{k}(\mathbf{W})=\left\|\mathbf{W}^{H} \mathbf{D}^{H} \mathbf{I}_{k}\right\|+\hat{\sigma}-\sqrt{1+\frac{1}{\gamma_{0}}} \cdot[\mathbf{D W}]_{k, k},
$$

where $\hat{\sigma}$ denotes the power of the equivalent noise $\hat{\mathbf{n}}$ before rescaling and can be obtained based on (48) as

$$
\hat{\sigma}=\sqrt{\frac{1}{\alpha^{2}}\left[\alpha^{2} \eta+\left(1-\alpha^{2}\right)+\sigma^{2}\right]} .
$$

Then, by substituting $\hat{A}_{i}(\mathbf{W})$ obtained in (51) into the optimization problems $\mathcal{P}_{3}$, the robust SINR balancing optimization with statistical CSI errors can be solved.

\section{B. Robust Design - Norm Bounded CSI Errors}

For completeness, we then consider the robust SINR beamforming with norm bounded CSI errors. Similar to the case of perfect CSI, we still construct the beamformer as

$$
\mathbf{P}=\frac{1}{\hat{f}} \cdot \mathbf{Z}^{-1} \hat{\mathbf{W}}
$$

Then, we note that the solution of SINR optimization for imperfect CSI is equivalent to solving a sequence of power minimization problems by the bisection search scheme [58][59]. Accordingly, in the following we focus on solving the corresponding robust power minimization problem for imperfect CSI, and the optimal beamforming vectors for SINR balancing can be obtained via the bisection search in Algorithm 1. With $\mathbf{P}$ given by (53), $\hat{f}=\left\|\mathbf{Z}^{-1} \hat{\mathbf{W}}\right\|$ is equal to the square root of the total transmit power for the corresponding 
power minimization problem. Then, we can express the power optimization problem as

$$
\begin{array}{ll}
\mathcal{P}_{8}: & \min _{\mathbf{z}_{L}, \hat{\mathbf{W}}} p \\
\text { s.t. } & p \geq\left\|\mathbf{Z}^{-1} \hat{\mathbf{W}}\right\|^{2} \\
& \frac{\left|\mathbf{d}_{k} \hat{\mathbf{w}}_{k}\right|^{2}}{\sum_{i \neq k}\left|\mathbf{d}_{k} \hat{\mathbf{w}}_{i}\right|^{2}+\sigma^{2}} \geq \gamma_{0}, \forall \mathbf{d}_{k} \in \mathcal{D}_{k}
\end{array}
$$

Note that the optimization problem $\mathcal{P}_{8}$ contains infinite number of constraints and is difficult to be directly solved. In the following we firstly assume that $\mathbf{z}_{L}$ is fixed and we focus on solving $\hat{\mathbf{W}}$, and the optimization on $\mathbf{z}_{L}$ directly follows Section III and IV. For the uncertainty region $\mathcal{D}_{k}$, we define the worst-case received SINR for user $k$ as

$$
\widetilde{S I N R_{k}}=\min _{\mathbf{d}_{k} \in \mathcal{D}_{k}} \frac{\left|\mathbf{d}_{k} \hat{\mathbf{w}}_{k}\right|^{2}}{\sum_{i \neq k}\left|\mathbf{d}_{k} \hat{\mathbf{w}}_{i}\right|^{2}+\sigma^{2}},
$$

and the optimization problem $\mathcal{P}_{8}$ is equivalent to

$$
\begin{array}{ll}
\mathcal{P}_{9}: & \min _{\hat{\mathbf{W}}} p \\
\text { s.t. } & p \geq\left\|\mathbf{Z}^{-1} \hat{\mathbf{W}}\right\|^{2} \\
& \widetilde{S I N R}_{k} \geq \gamma_{0}, \quad \forall \mathbf{d}_{k} \in \mathcal{D}_{k}
\end{array}
$$

By the definition of $\widetilde{S I N R}_{k}$ and $\mathcal{D}_{k}$, and by denoting

$$
\mathbf{T}_{k}=\frac{1}{\gamma_{0}} \hat{\mathbf{w}}_{k} \hat{\mathbf{w}}_{k}^{H}-\sum_{i \neq k} \hat{\mathbf{w}}_{i} \hat{\mathbf{w}}_{i}^{H}
$$

the constraint that $\widetilde{S I N R}{ }_{k} \geq \gamma_{0}$ can be further transformed into: for $\forall \mathbf{e}_{k} \mathbf{e}_{k}^{H} \leq \delta_{k}^{2}$,

$$
\begin{aligned}
& \mathbf{d}_{k} \mathbf{T}_{k} \mathbf{d}_{k}^{H}-\sigma^{2} \geq 0 \\
\Rightarrow & \left(\hat{\mathbf{d}}_{k}+\mathbf{e}_{k}\right) \mathbf{T}_{k}\left(\hat{\mathbf{d}}_{k}+\mathbf{e}_{k}\right)^{H}-\sigma^{2} \geq 0 \\
\Rightarrow & \mathbf{e}_{k} \mathbf{T}_{k} \mathbf{e}_{k}^{H}+\mathbf{e}_{k}\left(\mathbf{T}_{k} \hat{\mathbf{d}}_{k}^{H}\right)+\left(\mathbf{T}_{k} \hat{\mathbf{d}}_{k}^{H}\right)^{H} \mathbf{e}_{k}^{H}+\hat{\mathbf{d}}_{k} \mathbf{T}_{k} \hat{\mathbf{d}}_{k}^{H} \\
& -\sigma^{2} \geq 0
\end{aligned}
$$

Lemma: $\mathcal{S}$-procedure [60]: Let $\mathbf{T} \in \mathcal{C}^{N_{t} \times N_{t}}$ be a complex Hermitian matrix, $\mathbf{b} \in \mathcal{C}^{N_{t} \times 1}$ and a scalar $c$. For a vector $\mathbf{v} \in \mathcal{C}^{1 \times N_{t}}$, the following condition

$$
\mathbf{v} \mathbf{T} \mathbf{v}^{H}+\mathbf{v b}+\mathbf{b}^{H} \mathbf{v}^{H}+c \geq 0, \quad \forall\|\mathbf{v}\|^{2} \leq r^{2}
$$

is true if and only if there exists a non-negative value $t$ such that

$$
\mathbf{U}=\left[\begin{array}{cc}
\mathbf{T}+t \cdot \mathbf{I} & \mathbf{b} \\
\mathbf{b}^{H} & c-t \cdot r^{2}
\end{array}\right]
$$

is positive semi-definite.

Then, by employing the $\mathcal{S}$-procedure, (58) can be transformed into a positive semi-definite form, expressed as

$$
\left[\begin{array}{cc}
\mathbf{T}_{k}+t_{k} \cdot \mathbf{I} & \mathbf{T}_{k} \hat{\mathbf{d}}_{k}^{H} \\
\hat{\mathbf{d}}_{k} \mathbf{T}_{k}^{H} & \hat{\mathbf{d}}_{k} \mathbf{T}_{k} \hat{\mathbf{d}}_{k}^{H}-\sigma^{2}-t_{k} \delta_{k}
\end{array}\right] \succeq 0, \forall k \in \mathcal{K}
$$

where $t_{k} \geq 0$ is introduced as an auxiliary variable and $\mathcal{K}=$ $\{1,2, \ldots, K\}$. Then, by defining $\mathbf{X}_{k}=\hat{\mathbf{w}}_{k} \hat{\mathbf{w}}_{k}^{H},\left\|\mathbf{Z}^{-1} \hat{\mathbf{W}}\right\|^{2}$ can be transformed into

$$
\begin{aligned}
& \left\|\mathbf{Z}^{-1} \mathbf{W}\right\|^{2} \\
= & \operatorname{tr}\left\{\mathbf{Z}^{-1} \mathbf{W} \mathbf{W}^{H}\left(\mathbf{Z}^{-1}\right)^{H}\right\} \\
= & \operatorname{tr}\left\{\mathbf{Z}^{-1}\left[\hat{\mathbf{w}}_{1}, \hat{\mathbf{w}}_{2}, \ldots, \hat{\mathbf{w}}_{K}\right]\left[\hat{\mathbf{w}}_{1}, \hat{\mathbf{w}}_{2}, \ldots, \hat{\mathbf{w}}_{K}\right]^{H}\left(\mathbf{Z}^{-1}\right)^{H}\right\} \\
= & \sum_{k=1}^{K} \operatorname{tr}\left\{\mathbf{Z}^{-1} \mathbf{X}_{k}\left(\mathbf{Z}^{-1}\right)^{H}\right\} .
\end{aligned}
$$

Then, with (61) and (62), $\mathcal{P}_{9}$ can be recast as

$$
\begin{array}{ll}
\mathcal{P}_{10}: & \min _{\mathbf{X}_{k}, t_{k}} p \\
\text { s.t. } & p \geq \sum_{k=1}^{K} \operatorname{tr}\left\{\mathbf{Z}^{-1} \mathbf{X}_{k}\left(\mathbf{Z}^{-1}\right)^{H}\right\} \\
& \mathbf{T}_{k}=\frac{1}{\gamma_{k}} \mathbf{X}_{k}-\sum_{i \neq k} \mathbf{X}_{i}, \forall k \in \mathcal{K} \\
& {\left[\begin{array}{cc}
\mathbf{T}_{k}+t_{k} \cdot \mathbf{I} & \mathbf{T}_{k} \hat{\mathbf{d}}_{k}^{H} \\
\hat{\mathbf{d}}_{k} \mathbf{T}_{k}^{H} & \hat{\mathbf{d}}_{k} \mathbf{T}_{k} \hat{\mathbf{d}}_{k}^{H}-\sigma^{2}-t_{k} \delta
\end{array}\right] \succeq 0, \forall k \in \mathcal{K}} \\
& t_{k} \geq 0, \mathbf{X}_{k} \succeq 0, \operatorname{rank}\left(\mathbf{X}_{k}\right)=1, \forall k \in \mathcal{K}
\end{array}
$$

By dropping the rank constraint $\operatorname{rank}\left(\mathbf{X}_{k}\right)=1, \mathcal{P}_{10}$ becomes an SDP problem and can be efficiently solved with existing optimization tools. Furthermore, it has been shown in [56] that the optimal solution obtained always satisfies $\operatorname{rank}\left(\mathbf{X}_{k}^{*}\right)=1$, when the uncertainty bound $\delta_{k}$ is small. In this case, the exact optimal solution for the original problem $\mathcal{P}_{9}$ can be obtained by employing the eigenvalue decomposition. On the other hand, if $\operatorname{rank}\left(\mathbf{X}_{k}^{*}\right)>1$, randomization techniques in [61] or rank reduction approaches in [62] are widely adopted to obtain a feasible rank-one solution.

Specifically, for the conventional case where the mutual coupling matrix is fixed, we note that due to the fact that

$$
\mathbf{h}_{k}=\mathbf{d}_{k} \mathbf{Z}
$$

then based on (42) we have

$$
\begin{aligned}
\mathbf{h}_{k} & =\left(\hat{\mathbf{d}}_{k}+\mathbf{e}_{k}\right) \mathbf{Z}=\hat{\mathbf{h}}_{k}+\mathbf{e}_{k} \mathbf{Z} \\
& =\hat{\mathbf{h}}_{k}+\hat{\mathbf{e}}_{k},
\end{aligned}
$$

where $\hat{\mathbf{e}}_{k}$ denotes the channel error vector for fixed mutual coupling. Then, based on the sub-multiplicativity of the norm, we can further obtain

$$
\left\|\hat{\mathbf{e}}_{k}\right\|=\left\|\mathbf{e}_{k} \mathbf{Z}\right\| \leq\left\|\mathbf{e}_{k}\right\| \cdot\|\mathbf{Z}\| \leq \sqrt{\delta_{k}} \cdot\|\mathbf{Z}\|=\sqrt{\hat{\delta}_{k}} .
$$

Therefore, for the conventional case with fixed mutual coupling, the optimal beamforming vectors are obtained by solving $\mathcal{P}_{10}$ with $\hat{\delta}_{k}$ in (66), which guarantees that the SINR requirement is met.

Then, after the digital beamformers are obtained via the bisection search scheme with Algorithm 1, the optimization on $\mathbf{z}_{L}$ can be applied by incorporating $\mathcal{P}_{10}$ into the joint and decoupled beamforming scheme, respectively. For the joint beamforming scheme in Algorithm 2, $\hat{\mathbf{W}}^{(n+1)}$ within each 
iteration is obtained by solving $\mathcal{P}_{10}$ with the fixed mutual coupling matrix $\mathbf{Z}^{(n)}$ and $\delta_{k}$ in (43), where we note that an iterative design is still needed. As for the decoupled scheme, the digital beamforming vectors are firstly obtained by solving $\mathcal{P}_{10}$ with $\mathbf{Z}=\mathbf{I}$, and then the optimal load values that minimize the total transmit power are obtained by solving $\mathcal{P}_{6}$. In the case of quantized analog load values, the sequential search scheme of Section IV. B can be applied directly to the robust schemes here. With the above approaches, the robust SINR beamforming with mutual coupling exploitation for norm bounded CSI errors can be solved.

\section{Practical Implementation Aspects}

Based on the derivation of the proposed techniques, it can be observed that inline with typical beamforming techniques, the proposed schemes require the knowledge of CSI to perform the optimization. Throughout the derivation in Section III and IV we assume perfect CSI is known at the BS, while the extension to imperfect CSI is also discussed in the previous section. Moreover, it is observed that different from some beamforming schemes that require a symbol-by-symbol adaptation [14][18][63], the proposed optimization based beamforming schemes are independent of the transmit symbol vector.

Still, the proposed schemes require the adaptation of each load impedance $z_{L_{i}}$ dependent on the variation of the channels. Therefore, adaptive impedance tuning approaches are necessary. It has been shown in [64] and the references therein that varactor technologies that support adaptive impedance tuning can be divided into 3 categories: semiconductor-based varactor diodes, microelectromechanical system (MEMS) varactors, and ferroelectric-based varactors. Specifically, semiconductorbased varactor diodes and ferroelectric-based varactors can support the tuning speed as fast as 1-100 ns. An adaptive matching network is then introduced in [64] based on an automated impedance tuning unit with ferroelectric varactors, which can be applied to facilitate the proposed schemes. Moreover, the application of electronically steerable parasitic array radiators (ESPARs), where the radiation patterns are formed by changing the values of each load impedance [65][69] on a symbol-by-symbol basis, has further supported the implementation of the proposed schemes.

Therefore, based on the above the proposed schemes can be applied in practice and are mostly suitable for slow or quasistatic fading channels, where the channels change slowly.

\section{NumericAl RESUlts}

To evaluate the performance of the proposed beamforming schemes, in this section numerical results based on Monte Carlo simulations for the proposed schemes are presented. Since we have assumed $[\mathbf{D W}]_{i, i}>0, \forall i \in \mathcal{I}$ in (18), the obtained beamformers can be directly applied to evaluate the bit-error-rate (BER) performance. The system is operating at $2.6 \mathrm{GHz}$ and QPSK modulation is applied for the BER evaluation. The simulated channel is based on equations (4)(6) and the semi-correlated channel is assumed with $M=50$ DoDs and $\varphi=\pi / 8$. We assume a fixed antenna spacing at the transmitter and $d=0.25$ which is equivalent to $d=\lambda / 4$.
The initial load impedance vector $\mathbf{z}_{L}^{0}$ is $z_{L_{i}}=50 \Omega, \forall i \in$ $\left\{1,2, \ldots, N_{t}\right\}$. For the case of quantized load values, we assume $R_{\text {upper }}=100 \Omega, I_{\text {lower }}=-100 \Omega$, and $I_{\text {upper }}=100 \Omega$ respectively throughout the simulations. For the digital SINR balancing optimization, $\gamma_{\min }=0$ and $\gamma_{\max }$ is selected as $\gamma_{\max }=\frac{\max \left(\left\|\mathbf{h}_{k}\right\|^{2}\right)}{\sigma^{2}}$. The above parameters remain unchanged throughout the simulations unless otherwise stated. For clarity, the following abbreviations are used throughout the simulation results:

1) "SB with MC": conventional digital-only SINR balancing beamforming with fixed mutual coupling effect;

2) "SB no MC": conventional digital-only SINR balancing beamforming assuming no mutual coupling effect;

3) "SB A-D Joint": the proposed joint scheme for SINR balancing with continuous load values;

4) "SB A-D Decoupled": the proposed decoupled scheme for SINR balancing with continuous load values;

5) "SB A-D Sequential": the proposed sequential search scheme for SINR balancing with quantized load values;

6) "SB A-D Exhaustive": the exhaustive search scheme for SINR balancing with quantized load values.

For comparison in the numerical results we also present the simulation results for the ZF based scheme, where we note that ZF scheme can only be applied to the decoupled scheme, and the abbreviations for ZF based beamforming are denoted in a similar way.

\section{A. Perfect CSI}

Fig. 3 compares the BER performance of the proposed schemes and conventional digital schemes under perfect CSI. It is observed that for both ZF and SINR balancing, conventional schemes with fixed mutual coupling achieve the worst BER performance. For the proposed AD scheme, the joint beamforming achieves the best BER performance with the highest complexity, as the load impedances and the beamforming vectors are jointly optimized. For the decoupled approach,

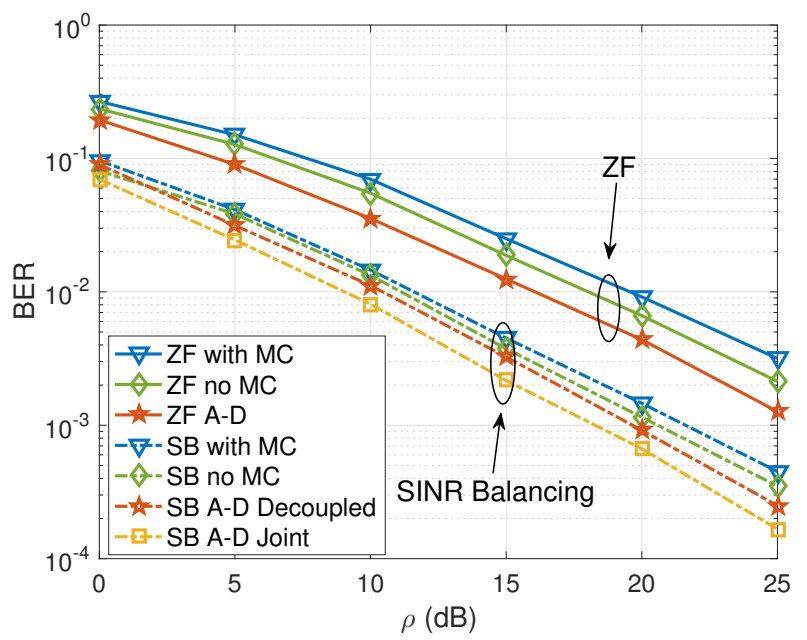

Fig. 3: BER with respect to the transmit SNR, $N_{t}=K=4$, QPSK, perfect CSI 


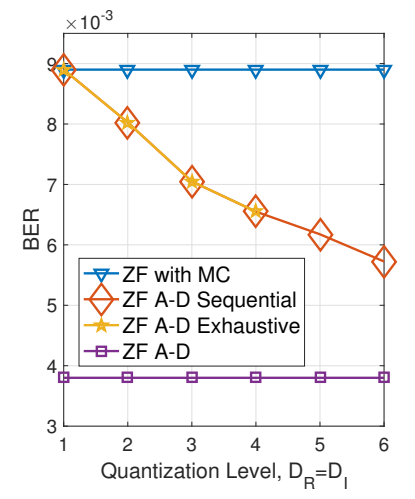

(a) ZF

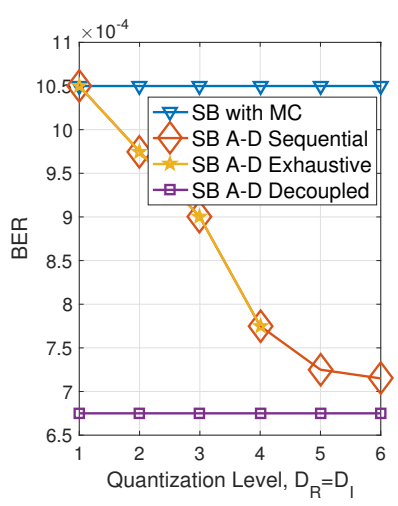

(b) SINR Balancing
Fig. 4: BER with respect to the quantization level, $N_{t}=K=$ 4, QPSK, perfect CSI, SNR=20dB

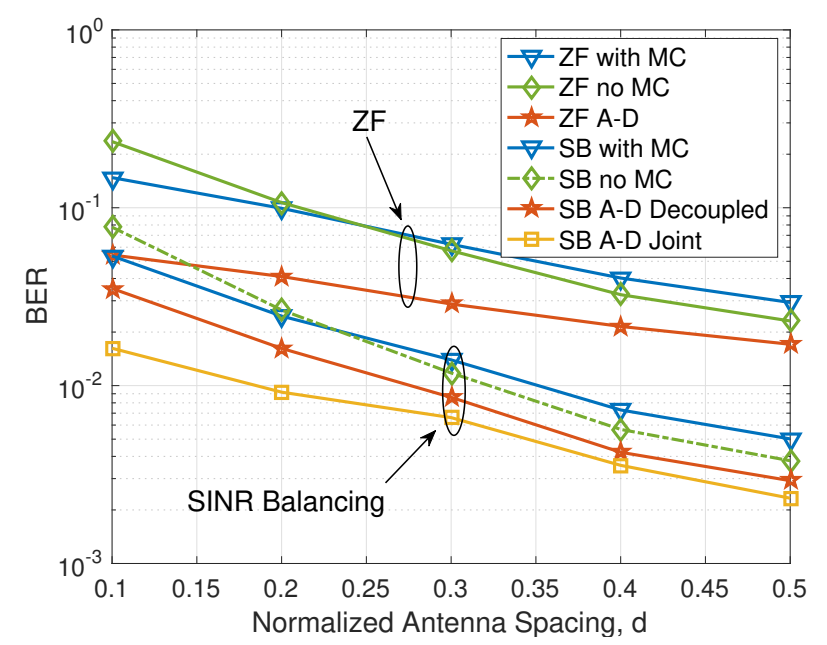

Fig. 5: BER with respect to the normalized antenna spacing, $N_{t}=K=4$, perfect CSI, $\mathrm{SNR}=10 \mathrm{~dB}$

while its performance is worse than the joint scheme, it is still superior to the conventional cases with fixed mutual coupling and without mutual coupling. Moreover, in line with existing results, beamforming schemes based on SINR balancing achieve better performance than ZF.

Fig. 4 shows the BER performance with respect to the quantization level at $\mathrm{SNR}=20 \mathrm{~dB}$ when quantized loads are employed, where for simplicity we assume $D_{R}=D_{I}$. When $D_{R}=D_{I}=1$, the case with quantized loads is identical to the case with fixed mutual coupling. For both ZF and SINR balancing beamforming, it is shown that mutual coupling can be beneficial with quantized tunable loads. More importantly, we have observed that for the considered scenario, the proposed sequential search scheme achieves a similar performance to the exhaustive search scheme, with greatly reduced complexity, where we note that the complexity for the exhaustive search is too high to simulate when the quantization level is larger than 4. It is also observed that with a larger quantization level and the resulting larger size of $\mathcal{S}$, the performance is improved and approaches the performance of the decoupled schemes.

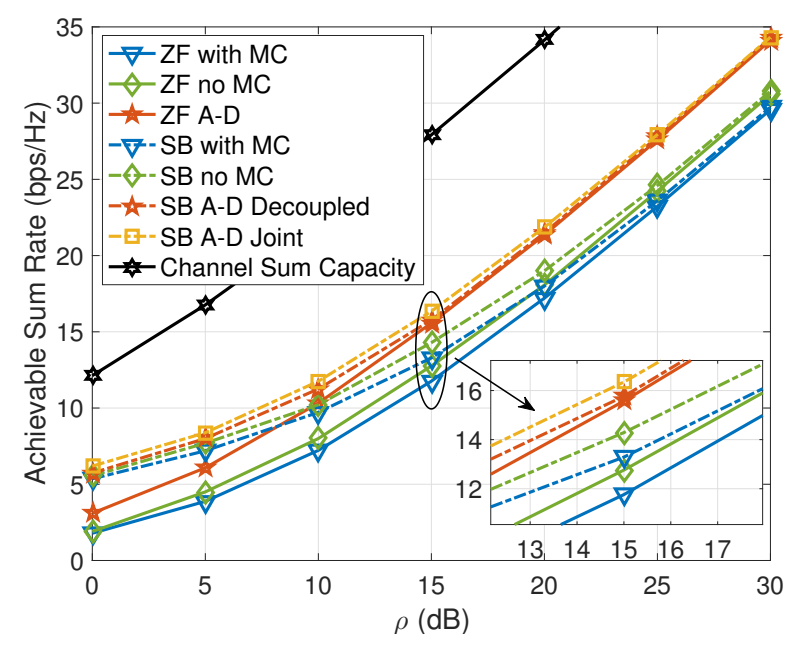

Fig. 6: Sum rate with respect to the transmit SNR, $N_{t}=K=$ 4, perfect CSI

Since the mutual coupling effect is highly relevant to the antenna spacing, in Fig. 5 we compare the BER performance with the increase in the normalized antenna spacing $d$. As can be observed, with a smaller antenna spacing, the performance gain of the proposed scheme with mutual coupling exploitation over the case with fixed mutual coupling is more significant, which is due to the increased mutual coupling effect that can be further exploited. Moreover, for all schemes, we have observed that the BER performance is improved with a larger antenna spacing, which is due to the reduced spatial correlation effect.

Fig. 6 shows the achievable sum rate for different beamforming schemes, where the channel sum capacity is given as

$$
C=\mathbb{E}\left\{\sup _{\mathbf{G} \in \mathcal{A}} \log _{2}\left[\operatorname{det}\left(\mathbf{I}+\frac{1}{\sigma^{2}} \mathbf{H}^{H} \mathbf{G H}\right)\right]\right\}
$$

where sup denotes the supremum function and $\mathcal{A}$ is the set of diagonal $K \times K$ matrices with nonnegative elements to ensure $\operatorname{tr}(\mathbf{G})=1$. When equal transmit power allocation is assumed, $\mathbf{G}=(1 / K) \cdot \mathbf{I}$. We note that $(67)$ is only employed to obtain the channel sum capacity for comparison, and the sum rate of the proposed schemes and ZF-based scheme can be obtained by (44) based on the received SINR. It can be seen from Fig. 6 that both the joint and the decoupled scheme offer a better rate performance compared to conventional digital beamforming with fixed mutual coupling. At low SNR, a rate gain as large as $7 \mathrm{~dB}$ can be observed for all SINR balancing based beamforming schemes over ZF schemes.

Fig. 7 shows the time complexity of the proposed joint scheme and the decoupled scheme, where we compare the average execution time of different beamforming schemes. For the joint beamforming scheme, $N_{\max }=5$. We observe that to obtain the beamformer, the joint scheme requires much more execution time than the decoupled scheme, and this gap becomes increasingly larger with the increase in the problem dimension. Nevertheless, it should be noted that the complexity of the joint scheme is mainly from the SINR 


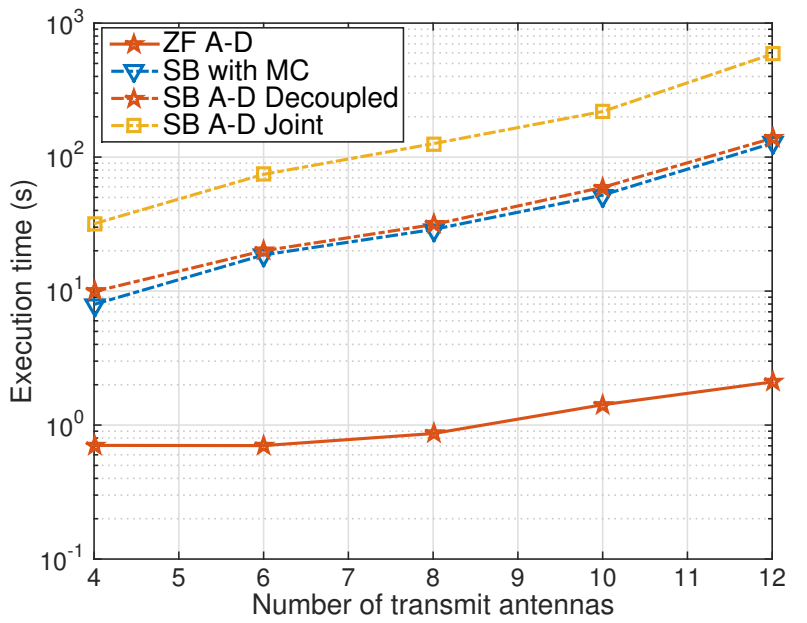

Fig. 7: Average execution time with respect to the number of antennas, $N_{t}=K, N_{\max }=5$

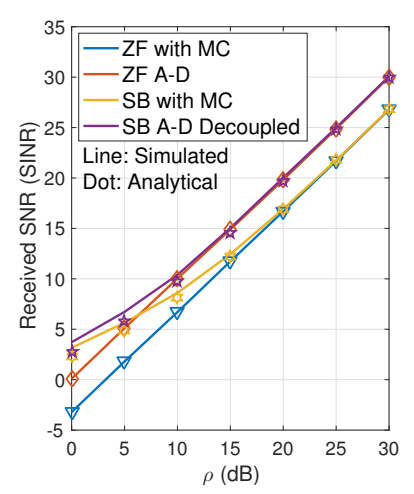

(a) Perfect CSI

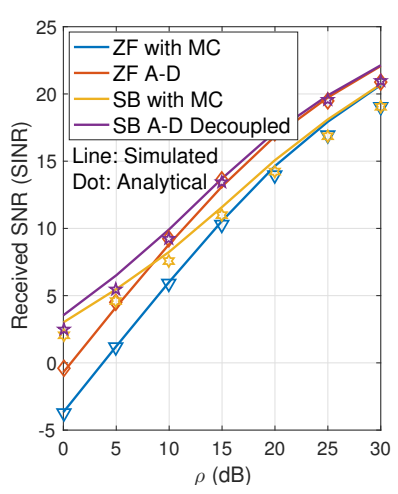

(b) Statistical CSI Errors
Fig. 8: Analytical results v.s. simulated results for $\mathrm{ZF}$ and SINR balancing, $N_{t}=K=4, \alpha=0.995, \beta=0.1$

balancing procedure, not from the optimization of the load impedances, as observed from the negligible gap between ' $\mathrm{SB}$ with MC' and 'SB A-D Decoupled'. It is also observed that 'ZF A-D' scheme requires the least complexity.

\section{B. Imperfect CSI - Statistical CSI Errors}

Next we present the results for imperfect CSI. We firstly consider the scenarios with statistical CSI errors, where without loss of generality we assume $\alpha=0.995$ and $\beta=0.1$. To validate our analysis of the received SINR, in Fig. 8 the analytical results and simulated results of the received SINR with perfect CSI and statistical CSI errors are shown respectively. As can be seen, for both the perfect and imperfect CSI cases, a close match between the analytical and simulated results can be observed.

Fig. 9 then shows the sum rate performance of the proposed robust scheme with statistical CSI errors. Compared to cases with perfect CSI, all beamforming schemes suffer a performance loss due to the channel estimation errors. At high SNR, due to the existence of the delay error matrix $\mathbf{Q}$,

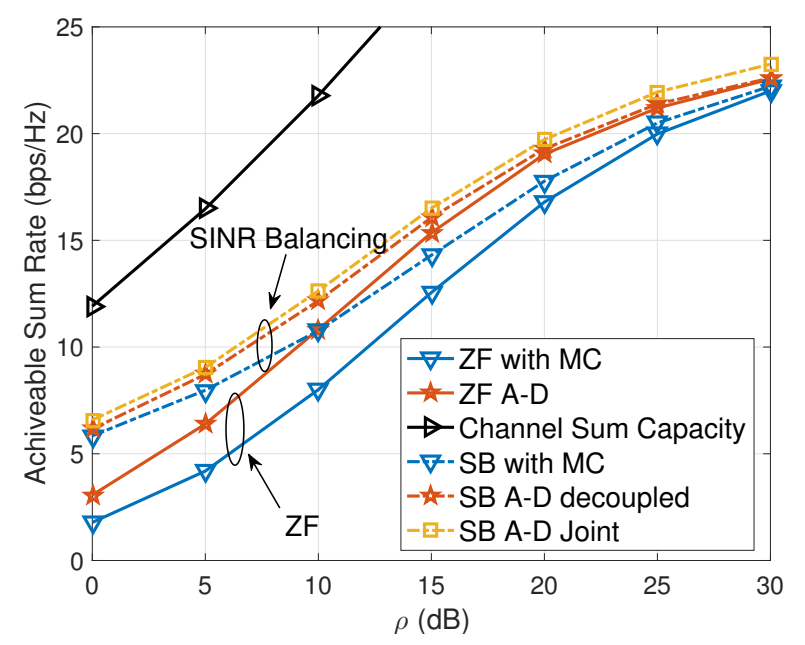

Fig. 9: Achievable sum rate of the robust SINR optimization, $N_{t}=K=4$, statistical CSI errors, $\alpha=0.995, \beta=0.1$

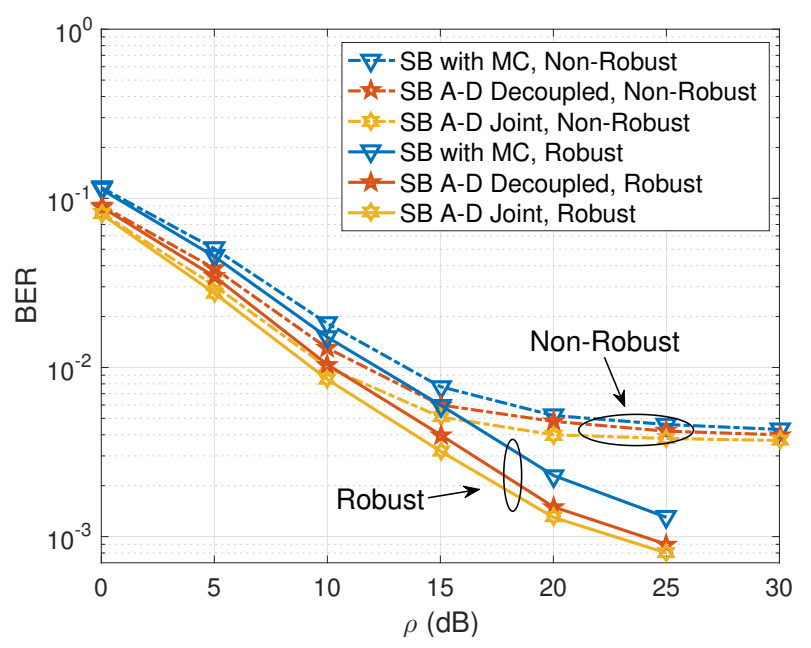

Fig. 10: BER with respect to the transmit SNR, $N_{t}=K=4$, QPSK, norm bounded CSI errors, $\delta_{k}=0.1$

the sum rate performance is upper bounded. More importantly, it is observed that by exploiting the mutual coupling effect, the proposed schemes still outperform the conventional digital beamforming schemes for the case of imperfect CSI, for both ZF and SINR balancing.

\section{Imperfect CSI - Norm Bounded CSI Errors}

We then proceed to present the simulation results with norm bounded CSI errors, where the channel error coefficient is assumed as $\delta_{k}=0.1, \forall k$. In Fig. 10, the BER performance is shown with respect to the increasing transmit SNR. As can be seen, compared to the non-robust scheme that is observed with an error bound at the high SNR regime, the robust scheme achieves an improved performance. Moreover, we observe that the proposed scheme based on exploiting the mutual coupling outperforms the conventional case with fixed mutual coupling. 


\section{CONCLUSION}

In this paper, a joint $\mathrm{AD}$ optimization based beamforming scheme that exploits the mutual coupling effect is proposed. We show that by judiciously picking the load values of each antenna element, the mutual coupling effect can be beneficial. Furthermore, for practical consideration of computational complexity and hardware imperfections, a lowcomplexity decoupled beamforming scheme and a sequential search scheme are proposed. For the case of imperfect CSI, the optimality is proven for closed-form beamforming schemes, and the robust SINR optimization scheme is further proposed. Simulation results show that compared to conventional cases where mutual coupling effect has a detrimental effect on the MIMO performance, the resulting mutual coupling with the proposed schemes further improves the system performance for both perfect CSI and imperfect CSI scenarios.

\section{APPENDIX \\ Calculation of the Antenna Impedance $z_{A}$ AND the MUTUAL IMPEDANCE $z_{m_{k}}$}

Based on [31], the antenna impedance $z_{A}$ and the mutual impedance $z_{m_{k}}$ can be calculated with EMF methods based on the antenna spacing $d$. For the antenna impedance, the real part and imaginary part can be calculated respectively as

$$
z_{A}=R_{z_{A}}+j \cdot X_{z_{A}}
$$

where $R_{z_{A}}$ is the resistance and $X_{z_{A}}$ is the reactance. The calculation of $R_{z_{A}}$ and $X_{z_{A}}$ is shown in (69) on the top of next page, where $\eta=120 \pi$ is the intrinsic impedance, $C_{i}$ and $S_{i}$ denote the cosine integral function and sine integral function, respectively. $\gamma_{0}$ is the Euler constant, $K=\frac{2 \pi}{\lambda_{0}}$, and $L=l \cdot \lambda_{0}$, where $\lambda_{0}$ is the carrier wavelength and $l$ is the length of the dipole antennas normalized by the carrier wavelength. $a$ is the radius of the wire and a typical value $a=0.001 \mathrm{~m}$ is applied in this paper. For half-wavelength dipole antennas, as employed in the simulation results, $l=0.5$ and the resulting $z_{A}=(73+j \cdot 42.5) \Omega$.

For a uniform linear half-wavelength dipole antenna array with side-by-side configuration, as assumed in this paper, the mutual impedance can be calculated as

$$
z_{m_{k}}=R_{m_{k}}+j \cdot X_{m_{k}} .
$$

In (70), the real part $R_{m_{k}}$ and imaginary part $X_{m_{k}}$ can be calculated respectively as

$$
\begin{aligned}
& R_{m_{k}}=\frac{\eta}{4 \pi}\left\{2 C_{i}\left[u_{0}(k)\right]-C_{i}\left[u_{1}(k)\right]-C_{i}\left[u_{2}(k)\right]\right\}, \\
& X_{m_{k}}=-\frac{\eta}{4 \pi}\left\{2 S_{i}\left[u_{0}(k)\right]-S_{i}\left[u_{1}(k)\right]-S_{i}\left[u_{2}(k)\right]\right\},
\end{aligned}
$$

where $u_{0}, u_{1}$ and $u_{2}$ can be calculated as

$$
\begin{aligned}
& u_{0}(k)=2 \pi \cdot k d, \\
& u_{1}(k)=2 \pi \cdot k\left(\sqrt{d^{2}+l^{2}}+l\right), \\
& u_{2}(k)=2 \pi \cdot k\left(\sqrt{d^{2}+l^{2}}-l\right),
\end{aligned}
$$

where $d$ is the normalized antenna spacing.

\section{REFERENCES}

[1] Evolved Universal Terrestrial Radio Access (E-UTRA): Physical Channels and Modulation. 3GPP TS 36.211, V 8.2.0, Release 8, March 2008.

[2] Evolved Universal Terrestrial Radio Access (E-UTRA): LTE Physical Layer; General Description. 3GPP TS 36.201, V.11.1.0, Release 11, March 2008.

[3] M. Costa, "Writing on Dirty Paper," IEEE Trans. Inf. Theory, vol. IT-29, no. 3, pp. 439-441, May 1983.

[4] C. Windpassinger, R. Fischer, T. Vencel, and J. Huber, "Precoding in Multiantenna and Multiuser Communications," IEEE Trans. Wireless Commun., vol. 3, no. 4, pp. 1305-1316, July 2004.

[5] L. Sun and M. Lei, "Quantized CSI-based Tomlinson-Harashima Precoding in Multiuser MIMO Systems," IEEE Trans. Wireless Commun. vol. 12, no. 3, pp. 1118-1126, March 2013.

[6] A. Garcia-Rodriguez and C. Masouros, "Power-Efficient TomlinsonHarashima Precoding for the Downlink of Multi-User MISO Systems," IEEE Trans. Commun., vol. 62, no. 6, pp. 1884-1896, April 2014.

[7] A. Li and C. Masouros, "A Constellation Scaling Approach to Vector Perturbation for Adaptive Modulation in MU-MIMO," IEEE Wireless Commun. Lett., vol. 4, no. 3, pp. 289-292, June 2015.

[8] B. M. Hochwald, C. B. Peel, and A. L. Swindlehurst, "A VectorPerturbation Technique for Near-Capacity Multiantenna Multiuser Communication-part II: Perturbation,” IEEE Trans. Commun., vol. 53, no. 3, pp. 537-544, March 2005.

[9] A. Li and C. Masouros, "A Two-Stage Vector Perturbation Scheme for Adaptive Modulation in Downlink MU-MIMO," IEEE Trans. Veh. Tech., vol. 65 , no. 9, pp. 7785-7791, Oct. 2015.

[10] C. Masouros, M. Sellathurai, and T. Ratnarajah, "Maximizing EnergyEfficiency in the Vector Precoded MU-MISO Downlink by Selective Perturbation," IEEE Trans. Wireless Commun., vol. 13, no. 9, pp. 4974 4984, Sept. 2014.

[11] R. Zi, X. Ge, J. Thompson, C.-X. Wang, H. Wang, and T. Han, "Energy Efficiency Optimization of 5G Radio Frequency Chain Systems," IEEE J. Sel. Areas Commun., vol. 34, no. 4, pp. 758-771, March 2016.

[12] T. Haustein, C. von Helmolt, E. Jorswieck, V. Jungnickel, and V. Pohl, "Performance of MIMO Systems with Channel Inversion," in Vehicular Technology Conference. IEEE 55th Vehicular Technology Conference. VTC Spring 2002, vol. 1, 2002, pp. 35-39.

[13] C. B. Peel, B. M. Hochwald, and A. L. Swindlehurst, "A VectorPerturbation Technique for Near-Capacity Multiantenna Multiuser Communication-part I: Channel Inversion and Regularization," IEEE Trans. Commun., vol. 53, no. 1, pp. 195-202, Jan. 2005.

[14] C. Masouros, "Correlation Rotation Linear Precoding for MIMO Broadcast Communications," IEEE Trans. Sig. Process., vol. 59, no. 1, pp. 252-262, Jan. 2011.

[15] F. Rashid-Farrokhi, K. Liu, and L. Tassiulas, "Transmit Beamforming and Power Control for Cellular Wireless Systems," IEEE J. Sel. Areas Commun., vol. 16, no. 8, pp. 1437-1450, Oct. 1998.

[16] M. Schubert and H. Boche, "Solution of the Multiuser Downlink Beamforming Problem with Individual SINR Constraints," IEEE Trans. Veh. Tech., vol. 53, no. 1, pp. 18-28, Jan. 2004.

[17] A. Wiesel, Y. C. Eldar, and S. Shamai (Shitz), "Linear Precoding via Conic Optimization for Fixed MIMO Receivers," IEEE Trans. Sig. Process., vol. 54, no. 1, pp. 161-176, Jan. 2006.

[18] C. Masouros and G. Zheng, "Exploiting Known Interference as Green Signal Power for Downlink Beamforming Optmization," IEEE Trans. Sig. Process., vol. 63, no. 14, pp. 3628-3640, July 2015.

[19] C. Masouros, M. Sellathurai, and T. Ratnarajah, "Large-Scale MIMO Transmitters in Fixed Physical Spaces: The Effect of Transmit Correlation and Mutual Coupling," IEEE Trans. Commun., vol. 61, no. 7, pp. 2794-2804, July 2013.

[20] C. Wang and R. D. Murch, "Adaptive Downlink Multi-User MIMO Wireless Systems for Correlated Channels with Imperfect CSI," IEEE Trans. Wireless Commun., vol. 5, no. 9, pp. 2435-2446, Sept. 2006.

[21] S. Biswas, C. Masouros, and T. Ratnarajah, "Performance Analysis of Large Multi-User MIMO Systems with Space-Constrained 2D Antenna Arrays," IEEE Trans. Wireless Commun., vol. 15, no. 5, pp. 3492-3505, May 2016.

[22] M. T. Ivrlac, W. Utschick, and J. A. Nossek, "Fading Correlations in Wireless MIMO Communication Systems," IEEE J. Sel. Areas Commun., vol. 21, no. 5, pp. 819-828, June 2003.

[23] A. M. Tulino, A. Lozano, and S. Verdu, "Impact of Antenna Correlation on the Capacity of Multiantenna Channels," IEEE Trans. Inf. Theory, vol. 51, no. 7, pp. 2491-2509, July 2005. 


$$
\begin{aligned}
R_{z_{A}}= & \frac{\eta}{2 \pi \sin ^{2}\left(\frac{K L}{2}\right)}\left\{\gamma_{0}+\ln (K L)-C_{i}(K L)+\frac{\sin (K L)}{2} \cdot\left[S_{i}(2 K L)-2 S_{i}(K L)\right]\right. \\
& \left.+\frac{\cos (K L)}{2}\left[\gamma_{0}+\ln \left(\frac{K L}{2}\right)+C_{i}(2 K L)-2 C_{i}(K L)\right]\right\}, \\
X_{z_{A}}= & \frac{\eta}{4 \pi \sin ^{2}\left(\frac{K L}{2}\right)}\left\{2 S_{i}(K L)+\cos (K L) \cdot\left[2 S_{i}(K L)-S_{i}(K L)\right]-\sin (K L) \cdot\left[2 C_{i}(K L)-C_{i}(2 K L)-C_{i}\left(\frac{2 K a^{2}}{L}\right)\right]\right\} .
\end{aligned}
$$

[24] G. Alfano, A. M. Tulino, A. Lozano, and S. Verdu, "Capacity of MIMO Channels with One-Sided Correlation," in Eighth IEEE International Symposium on Spread Spectrum Techniques and Applications - Programme and Book of Abstracts, 2004, pp. 515-519.

[25] M. Kiessling and J. Speidel, "Analytical Performance of MIMO ZeroForcing Receivers in Correlated Rayleigh Fading Environments," in 2003 4th IEEE Workshop on Signal Processing Advances in Wireless Communications - SPAWC 2003, 2003, pp. 383-387.

[26] H. Liu, Y. Song, and R. C. Qiu, "The Impact of Fading Correlation on the Error Performance of MIMO Systems over Rayleigh Fading Channels," IEEE Trans. Wireless Commun., vol. 4, no. 5, pp. 2014-2019, Sept. 2005.

[27] A. Alexiou and M. Qaddi, "Robust Linear Precoding to Compensate for Antenna Correlation in Orthogonal Space-Time Block Coded System," in Processing Workshop Proceedings, 2004 Sensor Array and Multichannel Signal, 2004, pp. 701-705.

[28] H. R. Bahrami and T. Le-Ngoc, "Precoder Design based on the Channel Correlation Matrices," IEEE Trans. Wireless Commun., vol. 5, no. 12, pp. 3579-3587, Dec. 2006.

[29] J. Akhtar and D. Gesbert, "Spatial Multiplexing over Correlated MIMO Channels with a Closed-Form Precoder," IEEE Trans. Wireless Commun., vol. 4, no. 5, pp. 2400-2409, Sept. 2005.

[30] B. Clerckx, C. Craeyo, D. V-Janvier, and C. Oestges, "Impact of Antenna Coupling on 2x2 MIMO Communications," IEEE Trans. Veh. Tech., vol. 56, no. 3, pp. 1009-1018, May 2007.

[31] D.-W. Seo and N.-H. Myung, "Analysis of Mutual Coupling Effects on Channel Capacity of Rician MIMO Channel," in 2008 Asia-Pacific Microwave Conference, Macau, 2008, pp. 1-4.

[32] A. A. Abouda and S. G. Haggman, "Effect of Mutual Coupling on Capacity of MIMO Wireless Channels in High SNR," Progress in Electromagnetics Research, vol. 65, pp. 27-40, 2005.

[33] C. A. Balanis, Antenna Theory: Analysis and Design, 4th ed. WileyBlackwell, March 2016.

[34] I. Gupta and A. Ksienski, "Effect of Mutual Coupling on the Performance of Adaptive Arrays," IEEE Trans. Ant. Propag., vol. 31, no. 5, pp. 785-791, Sept. 1983.

[35] J. W. Wallace and M. A. Jensen, "The Capacity of MIMO Wireless System with Mutual Coupling," in Proceedings IEEE 56th Vehicular Technology Conference, vol. 2, 2002, pp. 696-700.

[36] V. Jungnickel, V. Pohl, and C. von Helmolt, "Capacity of MIMO Systems with Closely Spaced Antennas," IEEE Commun. Lett., vol. 7, no. 8, pp. 361-363, Aug. 2003.

[37] X. Liu and M. E. Bialkovwski, "Effect of Antenna Mutual Coupling on MIMO Channel Estimation and Capacity," Int. J. Antennas Propag., pp. $1-9,2010$.

[38] T. Svantesson and A. Ranheim, "Mutual Coupling Effects on the Capacity of Multielement Antenna Systems," in 2001 IEEE International Conference on Acoustics, Speech, and Sigal Processing., vol. 4, 2001, pp. 2485-2488.

[39] H. Steyskal and J. S. Herd, "Mutual Coupling Compensation in Small Array Antennas," IEEE Trans. Ant. Propag., vol. 38, no. 2, pp. 19711975, Dec. 1990.

[40] J. Corcoles, M. A. Gonzalez, and J. Rubio, "Mutual Coupling Compensation in Arrays Using a Spherical Wave Expansion of the Radiated Field," IEEE Ant. Wireless Propag. Lett., vol. 8, pp. 108-111, Jan. 2009.

[41] S. Farsi, H. Aliakbarian, D. Schreurs, B. Nauwelaers, and G. A. E. Vandenbosch, "Mutual Coupling Reduction between Planar Antennas by Using a Simple Microstrip U-Section," IEEE Ant. Wireless Propag. Lett., vol. 11, pp. 1501-1503, Dec. 2012.

[42] G. Moreno, H. M. Bernety, and A. B. Yakovlev, "Reduction of Mutual Coupling between Strip Dipole Antennas at Terahertz Frequencies with an Elliptically Shaped Graphene Monolayer," IEEE Ant. Wireless Propag. Lett., vol. 15, pp. 1533-1536, Dec. 2015.

[43] J. Rubio, J. F. Izquierdo, and J. Corcoles, "Mutual Coupling Compensation Matrices for Transmitting and Receiving Arrays," IEEE Trans. Ant. Propag., vol. 63, no. 2, pp. 839-843, Feb. 2015.

[44] Z. Li, Z. Du, M. Takahashi, K. Saito, and K. Ito, "Reducing Mutual Coupling of MIMO Antennas with Parasitic Elements for Mobile Terminals," IEEE Trans. Ant. Propag., vol. 60, no. 2, pp. 473-481, Feb. 2012.

[45] A. Li and C. Masouros, "Exploiting Constructive Mutual Coupling is P2P MIMO by Analog-Digital Phase Alignment," IEEE Trans. Wireless Commun., vol. 16, no. 3, pp. 1948-1962, Mar. 2017.

[46] S. Han, C. I. I, and C. Rowell, "Large-Scale Antenna Systems with Hybrid Analog and Digital Beamforming for Millimeter Wave 5G," IEEE Commun. Mag., vol. 53, no. 1, pp. 186-194, Jan. 2015.

[47] A. Li and C. Masouros, "Hybrid Analog-Digital Millimeter-Wave MUMIMO Transmission with Virtual Path Selection," IEEE Commun. Lett., vol. 21, no. 2, pp. 438-441, Feb. 2017.

[48] — "Hybrid Precoding and Combining Design for Millimeter-Wave Multi-user MIMO based on SVD," in 2017 IEEE International Conference on Communications (ICC), Paris, 2017, pp. 1-6.

[49] A. Garcia-Rodriguez, C. Masouros, and P. Rulikowski, "Reduced Switching Connectivity for Large Scale Antenna Selection," IEEE Trans. Commun., vol. 65, no. 5, pp. 2250-2263, May 2017.

[50] J. Choma and W. K. Chen, Feedback Networks: Theory and Circuit Applications. U.S.A.: World Scientific Publishing, May 2007.

[51] H. A. Haus, Electromagnetic Noise and Quantum Optical Measurements. Springer, Nov. 2000.

[52] M. Razaviyayn, M. Hong, and Z.-Q. Luo, "A Unified Convergence Analysis of Block Successive Minimization Methods for Nonsmooth Optimization," SIAM J. Optim., vol. 23, no. 2, pp. 1126-1153, 2013.

[53] Q. Zhang, C. He, and L. Jiang, "Per-Stream MSE based Linear Transceiver Design for MIMO Interference Channels with CSI Error,' IEEE Trans. Commun., vol. 63, no. 5, pp. 1676-1689, May 2015.

[54] M. Ding and S. Blostein, "MIMO Minimum Total MSE Transceiver Design with Imperfect CSI at Both Ends," IEEE Trans. Sig. Process., vol. 57, no. 3, pp. 1141-1150, March 2009.

[55] J. Wang and D. Palomar, "Worst-Case Robust MIMO Transmission with Imperfect Channel Knowledge," IEEE Trans. Sig. Process., vol. 57, no. 8, pp. 3086-3100, Aug. 2009.

[56] E. Song, Q. Shi, M. Sanjabi, R. Sun, and Z.-Q. Luo, "Robust SINRConstrained MISO Downlink Beamforming: When is Semidefinite Programming Relaxation Tight?" in 2011 IEEE International Conference on Acoustics, Speech and Signal Processing (ICASSP), 2011, pp. 30963099.

[57] J. Maurer, J. Jalden, D. Seethaler, and G. Matz, "Vector Perturbation Precoding Revisited," IEEE Trans. Sig. Process., vol. 59, no. 1, pp. 315-328, Jan. 2011.

[58] F. Wang, X. Wang, and Y. Zhu, "Transmit Beamforming for Multiuser Downlink with Per-Antenna Power Constraints," in 2014 IEEE International Conference on Communications (ICC), Sydney, NSW, 2014, pp. 4692-4697.

[59] M. F. Hanif, L.-N. Tran, A. Tolli, and M. Juntti, "Computationally Efficient Robust Beamforming for SINR Balancing in Multicell Downlink with Applications to Large Antenna Array Systems," IEEE Trans. Commun., vol. 62, no. 6, pp. 1908-1920, June 2014.

[60] S. Boyd and L. Vandenberghe, Convex Optimization. Cambridge, U.K.: Cambridge University Press, 2004.

[61] Z.-Q. Luo, W. Ma, A. So, Y. Ye, and S. Zhang, "Semidefinite Relaxation of Quadratic Optimization Problems," IEEE Sig. Process. Mag., vol. 27, no. 3, pp. 20-34, May 2010. 
[62] Y. Huang and D. Palomar, "Rank-Constrained Separable Semidefinite Programming with Applications to Optimal Beamforming," IEEE Trans. Sig. Process., vol. 58, no. 2, pp. 664-678, Feb. 2010.

[63] S. M. Razavi and T. Ratnarajah, "Adaptively Regularized Phase Alignment Precoding for Multiuser Multiantenna Downlink," IEEE Trans. Veh. Tech., vol. 64, no. 10, pp. 4863-4869, Nov. 2014.

[64] J.-S. Fu, "Adaptive Impedance Matching Circuits based on Ferroelectric and Semiconductor Varactors," Ph.D. dissertation, Electrical Engineering, University of Michigan, 2009.

[65] A. Li, C. Masouros, and C. B. Papadias, "MIMO Transmission for Single-fed ESPAR with Quantized Loads," IEEE Trans. Commun., vol. 65, no. 7, pp. 2863-2876, July 2017.

[66] A. Li, C. Masouros, M. Sellathurai, and C. B. Papadias, "Tunable Load MIMO with Quantized Loads," in 2017 25th European Signal Processing Conference (EUSIPCO), Kos, 2017, pp. 1699-1703.

[67] L. Zhou, F. A. Khan, T. Ratnarajah, and C. B. Papadias, "Achieving Arbitrary Signals Transmission Using a Single Radio Frequency Chain," IEEE Trans. Commun., vol. 63, no. 12, pp. 4865-4878, Oct. 2015.

[68] G. C. Alexandropoulos, V. I. Barousis, and C. B. Papadias, "Precoding for Multiuser MIMO Systems with Single-Fed Parasitic Antenna Arrays," in 2014 IEEE Global Communications Conference (GLOBECOM), Dec. 2014, pp. 3897-3902.

[69] O. N. Alrabadi, C. Divarathne, P. Tragas, A. Kalis, N. Marchetti, C. B. Papadias, and R. Prasad, "Spatial Multiplexing with a Single Radio: Proof-of-Concept Experiments in an Indoor Environments with a 2.6GHz Prototype," IEEE Commun. Lett., vol. 15, no. 2, pp. 178-180, Feb. 2011.

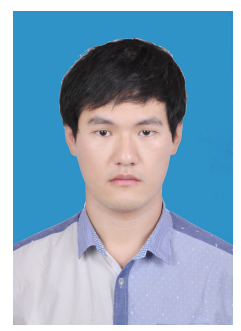

Ang Li (S'14) received the Bachelor and Master degree in Electronic and Information Engineering from Xi' an Jiaotong University in 2011 and 2014, respectively. He is currently pursuing the Ph.D. degree in the Communications and Information Systems research group, Dept. Electrical \& Electronic Engineering, University College London. His research interests lie in the field of wireless communications with focus on beamforming designs for MIMO systems and mmWave communications.

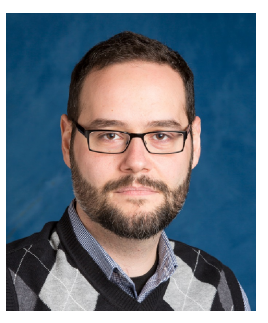

Christos Masouros (M'06-SM'14) received the Diploma degree in Electrical and Computer Engineering from the University of Patras, Greece, in 2004, and MSc by research and PhD in Electrical and Electronic Engineering from the University of Manchester, UK in 2006 and 2009 respectively. In 2008 he was a research intern at Philips Research Labs, UK. Between 2009-2010 he was a Research Associate in the University of Manchester and between 2010-2012 a Research Fellow in Queen's University Belfast. He has held a Royal Academy of Engineering Research Fellowship between 2011-2016.

$\mathrm{He}$ is currently a Senior Lecturer in the Communications and Information Systems research group, Dept. Electrical \& Electronic Engineering, University College London. His research interests lie in the field of wireless communications and signal processing with particular focus on Green Communications, Large Scale Antenna Systems, Cognitive Radio, interference mitigation techniques for MIMO and multicarrier communications. He was the recipient of the Best Paper Award in the IEEE GlobeCom conference 2015, and has been recognised as an Exemplary Editor for the IEEE Communications Letters, and as an Exemplary Reviewer for the IEEE Transactions on Communications. He is an Associate Editor for IEEE Communications Letters, and Guest Editor for IEEE Journal on Selected Topics in Signal Processing issue "Exploiting Interference towards Energy Efficient and Secure Wireless Communications".

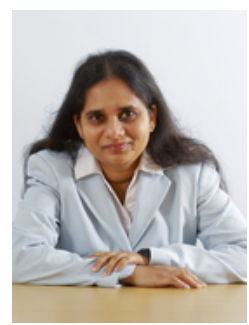

Mathini Sellathurai (S'95-M'02-SM'06) is a full Professor in Signal Processing and Intelligent Systems and Heriot-Watt University, Edinburgh, UK. In her 15-year research on Signal Processing for Communications, she has made seminal contributions on MIMO wireless systems. She has published 200 IEEE entries with $2300+$ citations, given invited talks and has written a book and several book chapters in topics related to this project. She received the IEEE Communication Society Fred W. Ellersick Best Paper Award in 2005, Industry Canada Public Service Awards for contributions in science and technology in 2005, and Best $\mathrm{PhD}$ thesis award (Silver Medal) from NSERC Canada in 2002. She is also a member for IEEE SPCOM Technical Strategy Committee, Editor for IEEE TSP (2009-2014, 2015-present). She is also the General Co-Chair of IEEE SPAWC2016 in Edinburgh. 